\title{
Article \\ Computational Investigation Identified Potential Chemical Scaffolds for Heparanase as Anticancer Therapeutics
}

\author{
Shraddha Parate ${ }^{1,+}\left(\mathbb{D}\right.$, Vikas Kumar ${ }^{2,+} \mathbb{C}$, Danishuddin ${ }^{2}$, Jong Chan Hong ${ }^{1, *}$ and Keun Woo Lee ${ }^{2, *}$ \\ 1 Division of Applied Life Science, Plant Molecular Biology and Biotechnology Research Center (PMBBRC), \\ Gyeongsang National University (GNU), 501 Jinju-daero, Jinju 52828, Korea; parateshraddha@gmail.com \\ 2 Division of Life Sciences, Department of Bio \& Medical Big Data (BK4 Program), Research Institute of Natural \\ Science (RINS), Gyeongsang National University (GNU), 501 Jinju-daero, Jinju 52828, Korea; \\ vikaspathania777@gmail.com (V.K.); danish.info16@gmail.com (D.) \\ * Correspondence: jchong@gnu.ac.kr (J.C.H.); kwlee@gnu.ac.kr (K.W.L.) \\ $\dagger$ These authors contributed equally.
}

Citation: Parate, S.; Kumar, V.; D.; Hong, J.C.; Lee, K.W. Computational Investigation Identified Potential Chemical Scaffolds for Heparanase as Anticancer Therapeutics. Int. J. Mol. Sci. 2021, 22, 5311. https://doi.org/ $10.3390 /$ ijms 22105311

Academic Editor: Giulio Vistoli

Received: 19 April 2021

Accepted: 17 May 2021

Published: 18 May 2021

Publisher's Note: MDPI stays neutral with regard to jurisdictional claims in published maps and institutional affiliations.

Copyright: (c) 2021 by the authors. Licensee MDPI, Basel, Switzerland. This article is an open access article distributed under the terms and conditions of the Creative Commons Attribution (CC BY) license (https:// creativecommons.org/licenses/by/ $4.0 /)$
Abstract: Heparanase (Hpse) is an endo- $\beta$-D-glucuronidase capable of cleaving heparan sulfate side chains. Its upregulated expression is implicated in tumor growth, metastasis and angiogenesis, thus making it an attractive target in cancer therapeutics. Currently, a few small molecule inhibitors have been reported to inhibit Hpse, with promising oral administration and pharmacokinetic (PK) properties. In the present study, a ligand-based pharmacophore model was generated from a dataset of well-known active small molecule Hpse inhibitors which were observed to display favorable PK properties. The compounds from the InterBioScreen database of natural $(69,034)$ and synthetic $(195,469)$ molecules were first filtered for their drug-likeness and the pharmacophore model was used to screen the drug-like database. The compounds acquired from screening were subjected to molecular docking with Heparanase, where two molecules used in pharmacophore generation were used as reference. From the docking analysis, 33 compounds displayed higher docking scores than the reference and favorable interactions with the catalytic residues. Complex interactions were further evaluated by molecular dynamics simulations to assess their stability over a period of $50 \mathrm{~ns}$. Furthermore, the binding free energies of the 33 compounds revealed 2 natural and 2 synthetic compounds, with better binding affinities than reference molecules, and were, therefore, deemed as hits. The hit compounds presented from this in silico investigation could act as potent Heparanase inhibitors and further serve as lead scaffolds to develop compounds targeting Heparanase upregulation in cancer.

Keywords: Heparanase; pharmacophore modeling; virtual screening; molecular docking; molecular dynamics simulations; binding free energy calculations; MM/PBSA

\section{Introduction}

The extracellular matrix (ECM) plays a major role in providing a physical scaffold for all the tissues and also helps to maintain the biochemical processes important for tissue homeostasis [1]. The supramolecular proteins (collagen, laminin, elastin, fibronectin) interact with the ubiquitous macromolecules heparan sulfate proteoglycans (HSPGs) in the cell surface and ECM, to maintain the cellular framework [2]. Heparanase (Hpse) (EC 3.2.1.166) is a principal endo- $\beta$-D-glucuronidase that catalyzes the cleavage of glycosaminoglycan heparan sulfate (HS) side chains of HSPGs into smaller fragments of 10-20 sugar units, thus modulating the HS function [3]. The mammalian Hpse enzyme was isolated from the placenta and later from platelets and its activity contributes to disassembly and remodeling of ECM and basement membrane [2,4]. Notably, these events result in upregulation of cell migration, invasion and release of HS-bound angiogenesis factors [2]. Markedly upregulated Hpse levels trigger the MMP-9, hepatocyte growth factor (HGF) and vascular endothelial growth factor (VEGF) expression, thereby leading to cancer progression [5-7]. The upregulation of Hpse expression levels has also been reported in numerous solid 
and hematological malignancies, including ovarian, pancreatic, brain, bladder, prostate, colon, liver, breast, sarcoma and myeloma [8,9]. Moreover, Hpse overexpression is also linked with aggressiveness of a variety of tumor cell types, and as observed clinically, its expression is also associated with increased tumor size, tumor progression enhancement, metastasis and poor prognosis [10]. These actions lead to pathological ailments, such as tissue inflammation and angiogenesis, therefore making Hpse a druggable target for developing anticancer therapeutics.

The pharmacological inhibition of Hpse overexpression has been accomplished through some HS mimetics, including Muparfostat (PI-88), Roneparstat (SST0001), Pixatimod (PG545) and Necuparanib (M402), which have entered clinical trials as Hpse inhibitors [11,12]. PI-88 (Figure S1) is a combination of sulfated di- to hexasaccharides and it progressed to the Phase III of clinical trials for hepatocellular carcinoma. However, it demonstrated hematologic side effects when administered along with Docetaxel [13,14]. Roneparstat (Figure S1), with an $\mathrm{IC}_{50}$ value in the nanomolar range, is an $\mathrm{N}$-acetylated glycol-split heparin and is in clinical trial for advanced multiple myeloma $[15,16]$. Pixatimod (Figure S1) is a fully sulfated hexasaccharide and a dual inhibitor of Hpse and angiogenesis, presently in Phase I of clinical trials for advanced solid tumors $[17,18]$. Necuparanib is a glycol-split HS mimetic and is currently in Phase-I/II clinical trials for metastatic pancreatic cancer when combined with gemcitabine and nab-paclitaxel $[19,20]$. Apart from the HS mimetic inhibitors, Zhao and co-workers identified a semisynthetic and highly sulfated oligosaccharide carbohydrate, JG3, from marine oligomannurarate [21]. JG3 inhibits both angiogenesis and metastasis and additionally blocks tumor growth. However, JG3 displays poor anticoagulant activity, and therefore, demonstrates low toxicity than other polyanionic compounds [1]. Another such marine-derived inhibitors for Hpse are a family of poly sulfated polygalactans originating from red algae and exhibiting anti-metastatic activity by inhibition of tumor-derived Hpse [22]. RK-682 (3-hexadecanoyl-5-hydroxymethyltetronic acid) (Figure S1), isolated from the mycelia of Streptomyces sp. 88-682, also displays an inhibitory activity for Hpse and the derivative 4-benzyl-RK-682 was also found to possess Hpse inhibitory activity ( $\mathrm{IC}_{50}$ $=17 \mu \mathrm{M})[23,24]$. Apart from the above-mentioned inhibitors, nucleic acid-based inhibitors such as Defibrotide have also been used to modulate the Hpse anti-cancer effect [1,25]. Defibrotide (Figure S1) is an orally bioavailable Hpse inhibitor, isolated from porcine intestinal mucosa, decreasing Hpse expression in multiple myeloma cell lines [26]. The developed Hpse inhibitors are predominantly carbohydrate-based compounds possessing heparin-like properties. However, these mimetics bind to heparin binding domains (HBD) flanking the Hpse active site, and therefore, are not specific for Hpse. Moreover, they interact with distinct heparin-binding proteins with off-target effects and unknown consequences [2]. Further disadvantages include their heterogeneous structures, which adds to their ambiguity as viable drugs for human use [2].

The discovery of small molecule Hpse inhibitors is desirable because of their efficient optimization for oral administration and promising pharmacokinetic properties, thereby resulting in an improved patient therapeutic compliance [27]. The polysulfonated naphthylurea-based small molecule, Suramin (Figure S1), inhibits melanoma Hpse and B16 melanoma cell invasion $[28,29]$. However, Suramin demonstrated adrenal insufficiency, neurotoxicity and renal toxicity along with anticoagulant-mediated blood dyscrasias, and therefore, failed to advance into clinical trials [1]. Additionally, several synthetic small molecules of various scaffolds have been reviewed in exclusive details by Mohan et al. [1], classifying them into benzazoles [27,30-32], thiazoles [33], oxazines [34-40], quinolines [41,42], glucans [41] and triazolo-thiadiazoles [2]. Apart from the inhibitors, Aspirin, which is a non-steroidal anti-inflammatory drug, was also found to inhibit Hpse by interacting with Glu225 in its catalytic site and observed to inhibit Hpse-mediated cancer cell migration, VEGF release and angiogenesis, both in vitro and in vivo [43].

Presently, a smaller quantity of small molecule inhibitors with promising pharmacokinetic properties are reported in literature for Hpse inhibition and the available HS mimetic inhibitors have failed at various stages of clinical trials. The search for new small 
molecule inhibitors with novel chemical scaffolds and the aforesaid perspectives prompted us to investigate natural as well as synthetic molecules as potential therapeutics targeted against Hpse. To attain this objective, we have carried out a ligand-based common-feature pharmacophore modeling study exploiting the shared chemical features of a few potent Hpse inhibitors, stated above. Accordingly, using the developed model as a query, we screened for compounds mapping our model, from a well-known InterBioScreen (IBS) database. We additionally checked their drug-likeness and performed molecular docking with the structure of Hpse. The acquired docked complexes were escalated further for evaluating their stability in physiological conditions. Subsequently, we subjected the simulated complexes to binding free energy calculations and confirmed two molecules each from natural and synthetic sources with better binding affinity than the reference compounds as hits.

\section{Results}

In the present in silico investigation, a ligand-based pharmacophore modeling approach employing a series of computational techniques have been applied for the identification of potential Hpse inhibitors. The schematic representation of the study is summarized as below (Figure 1).

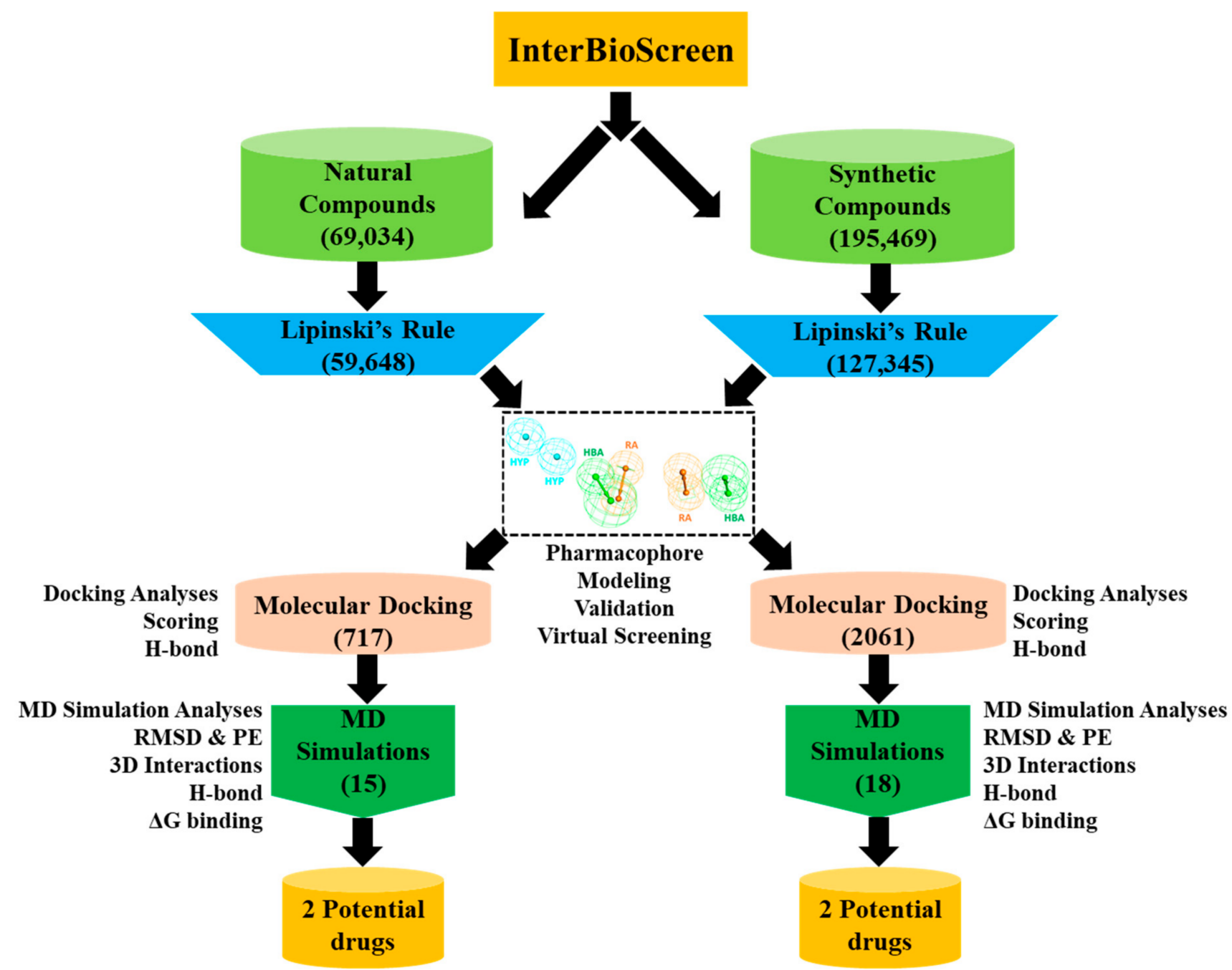

Figure 1. Flowchart depicting the working methodology in the current study used for the identification of potential Heparanase inhibitors. 


\subsection{Common Feature Pharmacophore Model}

The Feature Mapping protocol availed prior to model generation revealed the crucial ring aromatic (RA), hydrogen bond acceptor (HBA) and hydrophobic (HYP) features from four structurally diverse and well-known Hpse inhibitors as a training set (Figure 2), required for Hpse inhibition. Accordingly, the Common Feature Pharmacophore Generation module using the HipHop algorithm resulted in 10 model hypotheses with 5 or 6 features each. The rank of the generated models ranged from 65.96-71.08 (Table 1). For the evaluation of the ranks, features and alignment of inhibitors with the generated hypotheses, Hypo1 with the highest rank 71.08 was selected as the most reliable pharmacophore model. The model selected from the above step encompasses two RA, two HBA and two HYP features (Figure 3). The chosen model, Hypo1 was escalated for further validation by the Güner-Henry (GH) approach.

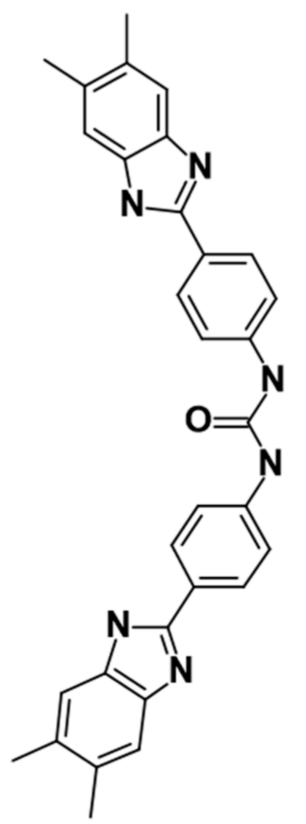

1 (0.07)

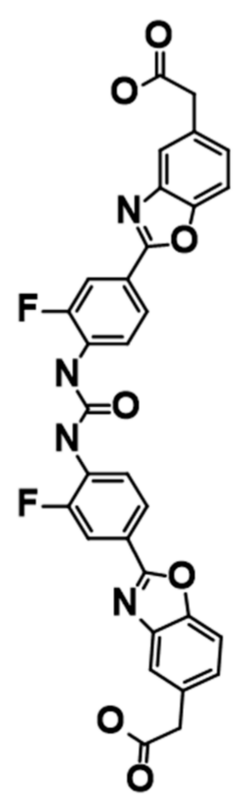

$2(0.18)$<smiles>O=C(O)Cc1ccc2oc(-c3ccc(/C=C/C(=O)Nc4ccc(Cl)c(Cl)c4)cc3F)nc2c1</smiles>

$3(0.20)$<smiles>COc1ccc(C(=O)Nc2ccc(CNc3ccc(-c4nc5ccccc5[nH]4)cn3)cc2)cc1Br</smiles>

4 (0.29)

Figure 2. Two-dimensional (2D) structures of four active compounds used as training set for pharmacophore hypotheses generation. The inhibitory activity value $\left(\mathrm{IC}_{50}\right)$ for each compound is shown in parentheses $(\mu \mathrm{M})$.

Table 1. Composition of the common-feature pharmacophore hypotheses generated by HipHop algorithm.

\begin{tabular}{|c|c|c|c|c|c|}
\hline Sr. No. & Features $^{a}$ & Rank $^{b}$ & Direct Hit $^{c}$ & Partial Hit $^{d}$ & Max Fit ${ }^{e}$ \\
\hline 1 & RA, RA, HBA, HBA, HYP, HYP & 71.08 & 1111 & 0000 & 6 \\
\hline 2 & RA, RA, HBA, HBA, HYP, HYP & 70.28 & 1111 & 0000 & 6 \\
\hline 3 & RA, RA, HBA, HBA, HYP, HYP & 70.28 & 1111 & 0000 & 6 \\
\hline 4 & RA, RA, HBA, HBA, HYP, HYP & 69.48 & 1111 & 0000 & 6 \\
\hline 5 & RA, HBA, HYP, HYP, HYPA & 66.18 & 1111 & 0000 & 5 \\
\hline 6 & HBA, HYP, HYP, HYPA, HYPA & 66.09 & 1111 & 0000 & 5 \\
\hline 7 & RA, HBA, HYP, HYP, HYPA & 66.04 & 1111 & 0000 & 5 \\
\hline 8 & RA, HBA, HYP, HYP, HYPA & 66.00 & 1111 & 0000 & 5 \\
\hline 9 & RA, RA, HBA, HYP, HYP & 65.96 & 1111 & 0000 & 5 \\
\hline 10 & RA, RA, HBA, HYP, HYP & 65.96 & 1111 & 0000 & 5 \\
\hline
\end{tabular}

${ }^{a}$ Features: RA: ring aromatic; HBA: hydrogen bond acceptor; HYP: hydrophobic; HYPA: hydrophobic aromatic. ${ }^{b}$ Rank: The best hypothesis demonstrates the highest rank. The higher the rank score, the lower the likelihood of chance correlation. ${ }^{\mathrm{c}}$ Direct Hit: Value (1) signifies that the training set molecules mapped well to all chemical features of the hypothesis. ${ }^{\mathrm{d}}$ Partial Hit: Value (0) signifies that there was no partial mapping of the training set molecules with the hypothesis. ${ }^{\mathrm{e}}$ Max Fit: The maximum number of features in the hypothesis. 


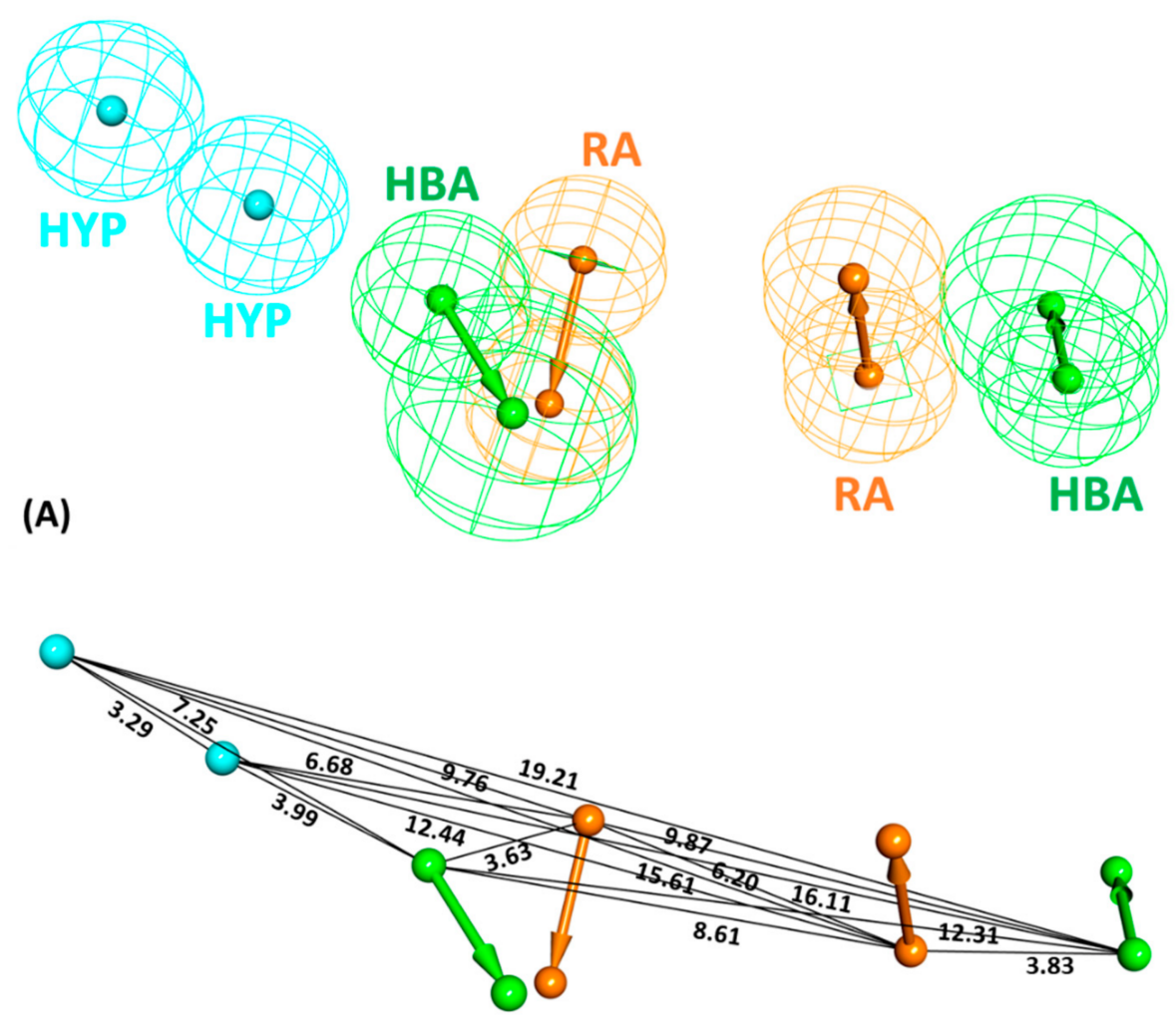

(B)

Figure 3. (A) The 3D representation of the selected pharmacophore model, Hypo1, containing two hydrophobic (HYP: cyan), two ring aromatic (RA: brown) and two hydrogen bond acceptor (HBA: green) features. (B) The interfeature distance $(\AA)$ among the features of Hypo1.

\subsection{Decoy Set Validation of the Pharmacophore Model}

The selected model, Hypo1, was assessed for its robustness in retrieving active Hpse molecules from a mixed database of active and decoy compounds. This decoy set validation was initiated by the DS Ligand Pharmacophore Mapping module, which retrieved the four inhibitors used in pharmacophore generation from a given external database of 100 compounds (4 active +96 decoys). Accordingly, the goodness of fit (GF) score was calculated as 0.72 , which was found near the ideal model range value of 1 (Table 2) [44,45]. The value of the GF score confirmed that our model is robust for further predicting active and potent Hpse compounds from an external database reasonably well.

Table 2. Decoy set validation of Hypo1 from an external database composed of active and inactive Heparanase inhibitors.

\begin{tabular}{ccc}
\hline Sr. No. & Parameters & Values \\
\hline 1 & Total number of compounds in the database (D) & 100 \\
2 & Total number of active compounds in the database $(\mathrm{A})$ & 4 \\
3 & Total number of hits retrieved by pharmacophore model & 6 \\
4 & from the database $(\mathrm{Ht})$ & 4 \\
5 & Total number of active compounds in the hit list $(\mathrm{Ha})$ & $66.66 \%$ \\
6 & $\%$ Yield of active ( $(\mathrm{Ha} / \mathrm{Ht}) \times 100)$ & $100 \%$ \\
7 & \% Ratio of actives $((\mathrm{Ha} / \mathrm{A}) \times 100)$ & 0 \\
8 & False negatives $(\mathrm{A}-\mathrm{Ha})$ & 2 \\
9 & False positives $(\mathrm{Ht}-\mathrm{Ha})$ & 0.72 \\
\hline
\end{tabular}




\subsection{Drug-Likeness Evaluation and Virtual Screening of InterBioScreen Database}

In the present in silico investigation, natural $(69,034)$ and synthetic $(195,469)$ compounds from the IBS database (Figure 1) were chosen for pharmacophore-based virtual screening. Prior to screening, a drug-like database of 186,993 compounds was prepared by Lipinski's rule of five (Ro5) from the above natural and synthetic compounds. Subsequently, the validated Hypo1 was used as a query to screen this drug-like database, mapping a total of 2778 compounds with the pharmacophoric features. The drug-like compounds derived from this virtual screening strategy were escalated for molecular docking with the Hpse molecular structure.

\subsection{Molecular Docking of Drug-Like Compounds with Heparanase}

The process of molecular docking depends on the prediction of the binding mode and the interaction with the catalytic site of a protein [46]. A total of 2778 compounds procured from virtual screening were subjected to docking with the GS3 Hpse model structure. Preceding this docking process, the GOLD software authenticity was checked by re-docking the bound ligand, resulting in an acceptable root mean square deviation (RMSD) of $0.78 \AA$ (Figure S2). Following the GOLD docking validation, the mapped compounds were docked with the Hpse molecular structure using the same coordinates utilized for bound ligand. Experimental studies revealed that molecules 1 and 2 (Figure 2) were reported to inhibit Hpse with the lowest inhibitory concentration. Therefore, to filter the true Hpse binding compounds, we considered both molecules as reference compounds and their docking scores as cutoff. Our docking analyses revealed that molecule 1 (REF1) displayed a Goldscore of 55.30 and a Chemscore of -27.79 , while molecule 2 (REF2) demonstrated a Goldscore of 67.43 and a Chemscore of -24.35 (Table S1). Further using these scores as cutoff, 15 natural and 18 synthetic compounds with higher docking scores and favorable interactions with the active site residues (Asp62, Asn64, Thr97, Glu225, Asn227, Lys231, Gln270, Arg272, Glu343, Gly349, Gly350, Ala388 and Tyr391) were selected (Table S1). These interactions were characterized by numerous bonds including hydrogen, hydrophobic and van der Waals. Finally, the above 33 complexes obtained from docking were escalated to molecular dynamics simulations for evaluating their stability.

\subsection{Molecular Dynamics Simulation Analysis}

The docked complexes of the 33 compounds with Hpse were taken as initial coordinates to check their stability at the atomistic level via molecular dynamics (MD) simulations. In total, 33 systems were prepared and subjected to simulation production run of $50 \mathrm{~ns}$ each, along with 2 simulation systems for REF inhibitors. Finally, the resulting MD simulation systems were ranked according to their binding free energies (BFE) with Hpse, via Molecular Mechanics Poisson-Boltzmann Surface Area (MM/PBSA) methodology (Table S1). The MM/PBSA results revealed that REF1 and REF2 displayed with BFE value of $-74.61 \mathrm{~kJ} / \mathrm{mol}$ and $-83.51 \mathrm{~kJ} / \mathrm{mol}$, respectively. Using the energy values of REF compounds as standard for the selection of potential inhibitors, we obtained four compounds with better BFE values, and therefore, these were considered as hit molecules (Table 3 and Table S2). Additionally, the stability of the selected hits was studied in terms of backbone RMSD, hydrogen bonds and potential energy plots. Furthermore, the binding interaction of the selected hits and REF compounds with the Hpse active site residues was scrutinized from the average structure taken from the last $5 \mathrm{~ns}$ of stable MD trajectories.

\subsubsection{Analysis of Stability and Binding Free Energy}

The backbone RMSD of simulated complexes was used to assess the stability and the systems were observed to be stable for the entire simulation period of $50 \mathrm{~ns}$ (Figure 4A,B). The hits acquired from the natural and synthetic IBS database presented with a convergent RMSD below $0.3 \mathrm{~nm}$ (Table 3). Concurrently, the BFE $\Delta \mathrm{G}_{\text {bind }}$ values were calculated by generating 50 frames from the entire simulation run and plotted accordingly (Figure 4C,D). The hits from natural compounds Hit1 (STOCK1N-70463) and Hit2 (STOCK1N-48729) from 
the IBS database displayed a BFE value of $-104.579 \mathrm{~kJ} / \mathrm{mol}$ and $-83.751 \mathrm{~kJ} / \mathrm{mol}$, respectively (Table 3 and Table S2, Figure 4C). Additionally, the hits from synthetic compounds Hit1 (STOCK1S-95244) and Hit2 (STOCK1S-71515) from the IBS database demonstrated a BFE value of $-96.193 \mathrm{~kJ} / \mathrm{mol}$ and $-86.806 \mathrm{~kJ} / \mathrm{mol}$, respectively (Table 3 and Table S2, Figure 4D). The BFE values of our hits were observed to be comparably better than the BFE values of the REF compounds stated above. Hence, from this analysis, it can be perceived that our natural and synthetic hits have better affinity towards Hpse. Moreover, the entire energy of all systems was observed to remain stable as seen from the potential energy plots for all complexes (Figure S3A,B). In addition to the RMSD, BFE and potential energy, the analysis of hydrogen bonds over the $50 \mathrm{~ns}$ simulation period suggested that our hits demonstrate a higher number of bonds throughout the simulation run (Figure S3C,D, Table 3).

Table 3. Molecular docking and molecular dynamics simulation analyses for reference (REF) inhibitors and selected potential hits from InterBioScreen (IBS) database against Heparanase.

\begin{tabular}{|c|c|c|c|c|c|}
\hline \multirow[b]{2}{*}{$\begin{array}{c}\text { Ligands } \\
\text { (IBS ID/REF No.) }\end{array}$} & \multicolumn{2}{|c|}{ Docking Scores } & \multicolumn{3}{|c|}{ MD Analyses } \\
\hline & Goldscore & Chemscore & $\begin{array}{c}\text { RMSD } \\
\text { (Backbone) }\end{array}$ & $\begin{array}{l}\text { Hydrogen Bond } \\
\text { (̊̊) }\end{array}$ & $\begin{array}{c}\text { Binding Free } \\
\text { Energy } \\
(\mathrm{kJ} / \mathrm{mol})\end{array}$ \\
\hline \multicolumn{6}{|c|}{ Natural Compound Hits } \\
\hline $\begin{array}{c}\text { Hit1 } \\
\text { (STOCK1N-70463) }\end{array}$ & 68.95 & -32.00 & 0.16 & 2.16 & $-104.579 \pm 20.649$ \\
\hline $\begin{array}{c}\text { Hit2 } \\
\text { (STOCK1N-48729) }\end{array}$ & 67.79 & -30.66 & 0.15 & 0.98 & $-83.751 \pm 26.469$ \\
\hline \multicolumn{6}{|c|}{ Synthetic Compound Hits } \\
\hline $\begin{array}{c}\text { Hit1 } \\
\text { (STOCK1S-95244) }\end{array}$ & 74.92 & -30.70 & 0.16 & 0.37 & $-96.193 \pm 23.866$ \\
\hline $\begin{array}{c}\text { Hit2 } \\
\text { (STOCK1S-71515) }\end{array}$ & 67.53 & -33.38 & 0.14 & 1.17 & $-86.806 \pm 26.536$ \\
\hline \multicolumn{6}{|c|}{ Reference Inhibitors } \\
\hline REF1 & 55.30 & -24.35 & 0.14 & 0.25 & $-74.612 \pm 20.900$ \\
\hline REF2 & 67.43 & -24.35 & 0.15 & 1.13 & $-83.519 \pm 31.504$ \\
\hline
\end{tabular}

2.5.2. Binding Mode and Molecular Interactions with Heparanase Active Site

The binding mode and interaction of our hits was scrutinized in detail by calculating the average structure from the last $5 \mathrm{~ns}$ of simulation run for each Hpse compound complex.

The inhibitor REF1 was observed to display one hydrogen bond with catalytic residue Glu225. In addition, REF1 formed hydrophobic bonds with residues Tyr298 ( $\pi$ - $\pi$ T-shaped), Val384 (alkyl) and Tyr391 ( $\pi$-alkyl). REF1 also formed bonds with Thr60, Asp62, Gly95, Gly96, Thr97, Ser228, Arg272, His296, Glu343, Gln383 and Ala388, characterized by van der Waals interactions (Figure S4A,C, Table 4). The binding of inhibitor REF2 was rendered by hydrogen bonds with residue Gly349 and hydrophobic bonds with residues- Arg272 ( $\pi$-cation) and Tyr391 ( $\pi$ - $\pi$ T-shaped). The Hpse catalytic residues Thr97, Gln270, Pro271, Tyr348, Gly350, Gln383, Gly389 and Asn390 form van der Waals interactions with REF2 (Figure S4B,D, Table 4). 
Natural-Compounds

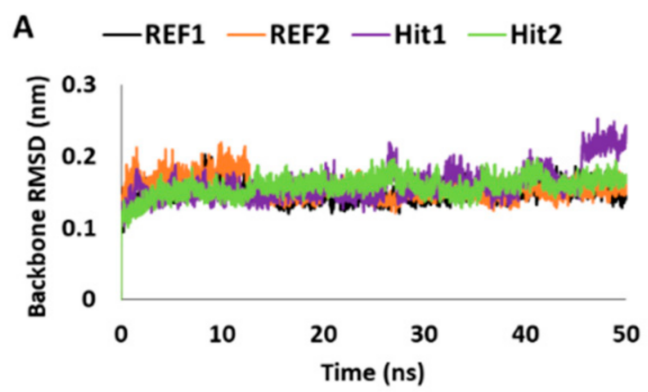

C

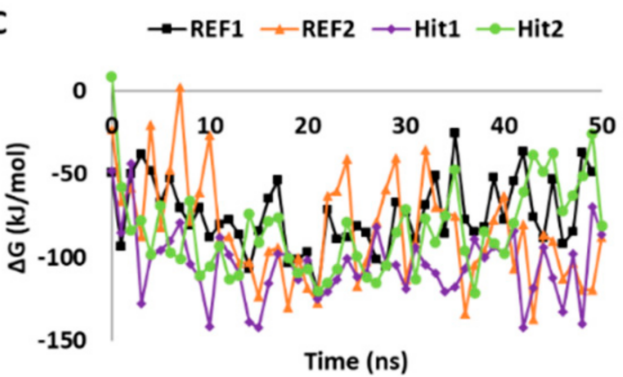

Synthetic-Compounds

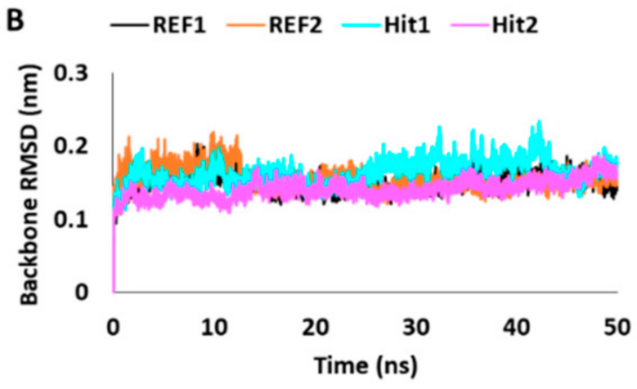

D

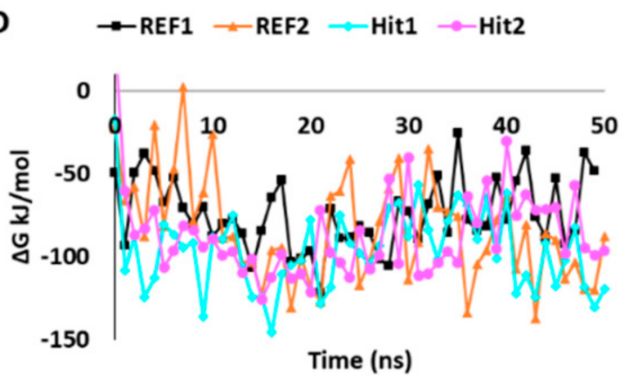

Figure 4. Molecular dynamics simulation analysis plots of Heparanase with the reference (REF) and Hits displaying $(\mathbf{A}, \mathbf{B})$ the backbone root mean square deviation (RMSD) and (C,D) the binding free energy $\left(\Delta G_{\text {bind }}\right)$ values. The left $(\mathbf{A}, \mathbf{C})$ and right $(\mathbf{B}, \mathbf{D})$ columns represent the analysis for natural and synthetic compound hits, respectively.

The average structure of Hit1, acquired from natural compounds, revealed that Hit1 demonstrated hydrogen bonds with Gln270, Asn227, Gly349 and Gly350 and hydrophobic interactions with Glu225 ( $\pi$-anion), Arg272 ( $\pi$-cation), Tyr348 ( $\pi-\pi$ T-shaped) and Tyr391 ( $\pi$-sigma). Moreover, residues Thr97, Ser228, Lys274, Thr275, Tyr298 and Gln383 hold Hit1 in the Hpse catalytic pocket via van der Waals interactions (Figure 5A,C, Table 4). The identified Hit2 obtained from natural compounds was observed to interact with Asn227, Tyr298 and Gly349 via hydrogen bonds. Hit2 additionally formed bonds with Arg272 ( $\pi$-cation) and Tyr348 ( $\pi-\pi$ T-shaped) via hydrophobic interactions. Residues Thr97, Gln270 and Lys274 hold Hit2 in the Hpse active site via van der Waals bonds (Figure 5B,D, Table 4).

The representative structure of Hit1 attained from virtual screening of synthetic compounds displayed hydrogen bonds with Hpse catalytic residues Asn227, Ser228 and Lys274. Hit1, moreover, formed $\pi$-alkyl hydrophobic bonds with Lys231, Lys232, Lys274 and Met278 and van der Waals interactions with Gln270 and Thr275 (Figure 6A,C, Table 4). Furthermore, Hit2 obtained from synthetic compounds exhibited hydrogen bonds with Gln270 and hydrophobic bonds with Gly349 (amide $\pi$-stacked) and Tyr391 ( $\pi$-alkyl). Additionally, Hit2 was supported by Thr97, Lys231, Pro271, His297, Tyr348, Gly350 and Gln383 via van der Waals interactions (Figure 6B,D, Table 4).

Overall, from the MD simulation analyses explained above, our identified hits display stability throughout the $50 \mathrm{~ns}$ of simulation run and also demonstrate comparably better binding affinities than REF inhibitors as observed from their BFE values. Moreover, the proposed hits in this study demonstrate intermolecular interactions with the key residues of Hpse catalytic site. Therefore, we anticipate that our hits can be deemed a good fit for Hpse inhibition. 
Table 4. Molecular interactions of the compounds (reference and hits) with Heparanase active site residues obtained from stable molecular dynamics simulation trajectories.

\begin{tabular}{|c|c|c|c|c|c|c|c|}
\hline \multirow{2}{*}{\multicolumn{2}{|c|}{ Complex Name }} & \multicolumn{4}{|c|}{ Hydrogen Bond Interactions } & \multirow{3}{*}{ van der Waals Interactions } & \multirow{3}{*}{$\pi-\pi / \pi$-alkyl Interactions } \\
\hline & & Amino Acid & Amino Acid Atom & Ligand Atom & Distance (<3.05 ̊̊) & & \\
\hline \multicolumn{6}{|c|}{ Natural Compound Hits } & & \\
\hline \multirow{6}{*}{$\begin{array}{c}\text { Heparanase } \\
+ \\
\text { Natural } \\
\text { Compounds }\end{array}$} & \multirow{3}{*}{ Hit1 } & $\mathrm{G} \ln 270$ & $\mathrm{HN}$ & $\mathrm{O} 13$ & 2.08 & \multirow{3}{*}{$\begin{array}{l}\text { Thr97, Ser228, Gly269, } \\
\text { Arg272, Lys274, Thr275, } \\
\text { Tyr298, Tyr348, Gln383 }\end{array}$} & \multirow{3}{*}{ Glu225, Tyr391 } \\
\hline & & Gly349 & $\mathrm{HN}$ & $\mathrm{O} 18$ & 1.90 & & \\
\hline & & Gly350 & $\mathrm{HN}$ & $\mathrm{O} 18$ & 2.29 & & \\
\hline & \multirow{3}{*}{ Hit2 } & Asn227 & HD21 & $\mathrm{O} 29$ & 2.07 & \multirow{3}{*}{$\begin{array}{l}\text { Thr97, Gln270, Lys274, } \\
\text { Thr275, Gly350 }\end{array}$} & \multirow{3}{*}{ Arg272, Tyr348 } \\
\hline & & Tyr298 & $\mathrm{HH}$ & O16 & 1.80 & & \\
\hline & & Gly349 & $\mathrm{HN}$ & O19 & 2.61 & & \\
\hline \multicolumn{8}{|c|}{ Synthetic Compound Hits } \\
\hline \multirow{4}{*}{$\begin{array}{c}\text { Heparanase } \\
+ \\
\text { Synthetic } \\
\text { Compounds }\end{array}$} & \multirow{3}{*}{ Hit1 } & Asn227 & $\mathrm{O}$ & H35 & 1.85 & \multirow{3}{*}{ Gln270, Arg272, Thr275 } & \multirow{3}{*}{ Lys231, Lys232, Met278 } \\
\hline & & Ser228 & HG & N6 & 2.68 & & \\
\hline & & Lys274 & HZ2 & $\mathrm{O} 15$ & 2.68 & & \\
\hline & Hit2 & $\mathrm{Gln} 270$ & HE21 & $\mathrm{O} 15$ & 2.60 & $\begin{array}{l}\text { Thr97, Lys231, Pro271, } \\
\text { His297, Tyr298, Tyr348, } \\
\text { Gly350, Gln383 }\end{array}$ & Gly349, Tyr391 \\
\hline \multicolumn{8}{|c|}{ Reference (REF) Inhibitors } \\
\hline \multirow{2}{*}{$\begin{array}{c}\text { Heparanase } \\
+ \\
\text { Reference } \\
\text { Inhibitors }\end{array}$} & REF1 & Glu225 & OE2 & H66 & 1.74 & $\begin{array}{c}\text { Thr60, Asp62, Gly95, Gly96, } \\
\text { Thr97, Ser228, Arg272, } \\
\text { His296, Glu343, } \\
\text { Gln383, Ala388 }\end{array}$ & Tyr298, Val384, Tyr391 \\
\hline & REF2 & $G \ln 349$ & $\mathrm{HN}$ & $\mathrm{O} 23$ & 2.39 & $\begin{array}{l}\text { Thr97, Gln270, Pro271, } \\
\text { Tyr348, Gly350, Gln383, } \\
\text { Gly389, Asn390 }\end{array}$ & Arg272, Tyr391 \\
\hline
\end{tabular}


A

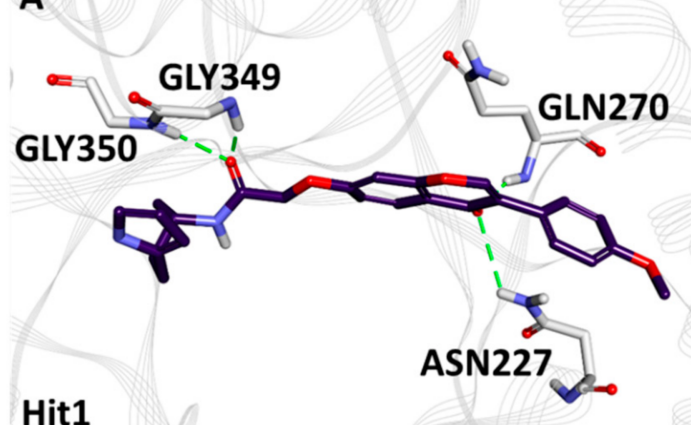

C

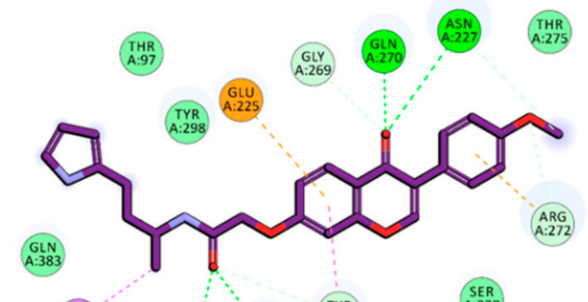

(TI39)

Hit1

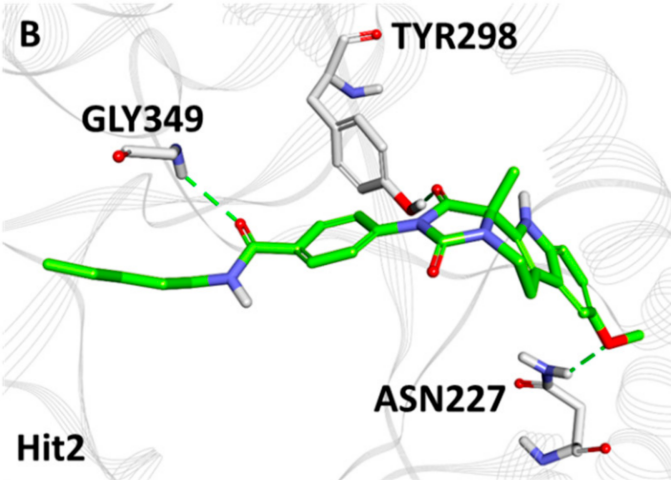

D

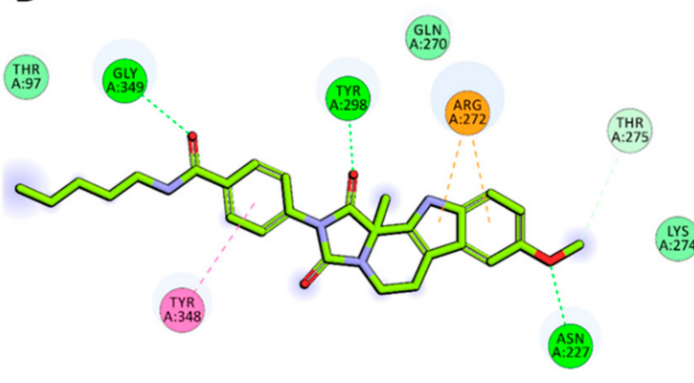

Hit2

Figure 5. The 3D and 2D intermolecular interactions of natural compound hits (Hit1: A,C; Hit2: B,D) with the active site residues of Heparanase.

A

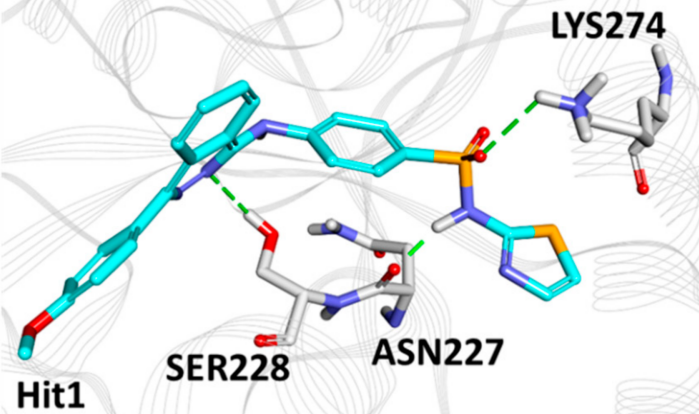

C

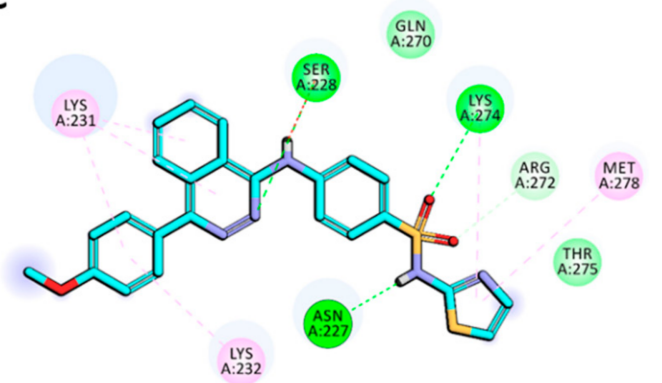

Hit1
B

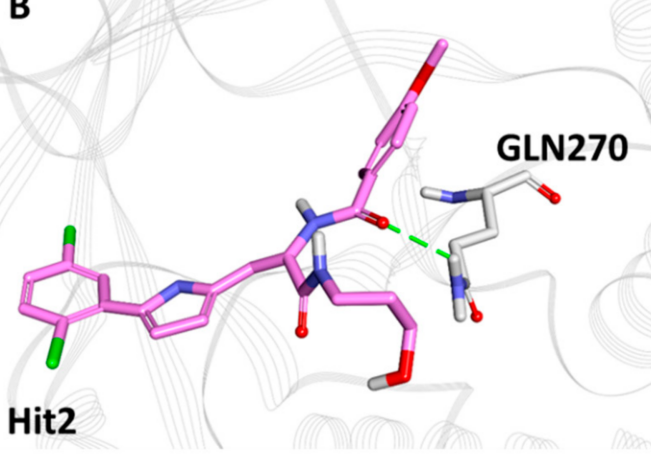

D

(1.25)

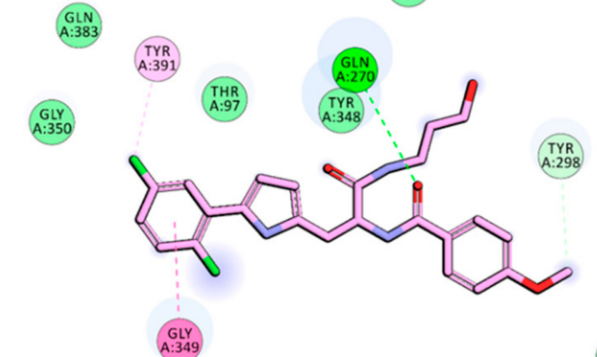

Hit2

(4:15)

Figure 6. The 3D and 2D intermolecular interactions of synthetic compound hits (Hit1: A,C; Hit2: B,D) with the active site residues of Heparanase. 


\section{Discussion}

Human Hpse is an endoglucuronidase that cleaves HS side chains, an indispensable component of the ECM. This event leads to remodeling of the ECM, causing a release of growth factors and cytokines bound to HS. The release of growth factors further promotes pathological processes, including angiogenesis, migration of immune cells, inflammation and metastasis. Quintessentially, all cancers examined to date have been reported to upregulate Hpse activity, instigating tumor growth and metastasis with poor patient survival [47]. Therefore, Hpse has emerged as a valid druggable target for developing effective anti-cancer therapeutics. The advent of Hpse inhibitors has resulted in the development of carbohydrate-based molecules with heparin-mimicking properties [11,25,48]. These heparin mimics bind to heparin-binding domains flanking the Hpse catalytic site, thereby inhibiting HS cleavage. Four such heparin mimetics, Roneparstat, Necuparanib, Mupafostat and Pixatimod, are presently in clinical trials for different cancerous ailments. However, mimetic inhibitors result in off-target effects and are not Hpse-specific, causing them to bind with heparin-binding protein domains [2]. Such mimics, additionally, are heterogeneous in their structures (both in composition and chain length), further adding to their vagueness as viable Hpse drugs for human use. These effects limit their standardization, product characterization, biological data interpretation, and may also affect their delivery route [27]. The discovery and development of small molecule Hpse inhibitors is particularly desirable owing to their satisfactory pharmacokinetic properties and optimization, leading to oral administration. Although remarkable progress has been made in the development of small molecule Hpse inhibitors, no drugs able to modulate its activity has reached the clinical setting. Taking into account the aforementioned viewpoints, we pursued our research objective to identify both natural as well as synthetic small molecules as Hpse inhibitors by applying Catalyst/HipHop-based common-feature pharmacophore modeling.

The Catalyst pharmacophore modeling program queries interactions of compounds with the target protein on the basis of two hypothesis generation methods, including HypoGen and HipHop [49,50]. The HypoGen method illuminates the correlation between the chemical binding features of the compounds and their biological activities. The HipHop method, on the other hand, focuses on the chemical features which are common to the dataset of the most active compounds with a narrow activity range [51]. This hypothesis generation method produces pharmacophore models independent of the in vitro biological activity of the training set compounds [52,53]. Therefore, the objective of the present study was to acquire the active Hpse inhibitors with unique scaffolds encompassing acetic acid, benzamide, urea and benzazolyl derivatives for developing a pharmacophore model to inhibit the upregulation of Hpse (Figure 2).

The chemical features of the aforementioned active inhibitors were exploited by the Catalyst/HipHop program, which generated 10 pharmacophore hypotheses with distinct ranks and features (Table 1). Among the 10 generated models, the best 3D pharmacophore model with a ranking score of 71.08 was selected, composed of two ring aromatic, two hydrogen bond acceptor and two hydrophobic as quintessential features, required for Hpse inhibition (Figure 3). Our findings were also in accordance with a previously published study by Gozalbes et al., where a four-point pharmacophore model was developed consisting of hydrogen bond donor, acceptor and hydrophobic features as most essential for Hpse inhibition [42]. Gozalbes et al. and team successfully identified the anti-malarial drug, Amodiaquine displaying Hpse inhibitory activity employing their pharmacophore model [41]. Therefore, we argue that our pharmacophore model, generated with similar features as previous studies, gives reliable results for retrieving compounds that display a better binding affinity towards Hpse. The chosen Hpse pharmacophore model with the highest rank score was further validated by the decoy set validation method, generating a GF score of 0.72 , which is near the ideal model range value of 1 (Table 2) [53-55]. The validated model was, thus, considered to be robust for retrieving molecules from an external database, and therefore, the resultant model was allowed to screen the InterBioScreen database composed of natural $(69,034)$ and synthetic $(195,469)$ compounds (Figure 1). Prior 
to virtual screening, the total number of molecules from both the subsets was reduced to 59,649 (natural) and 127,345 (synthetic) drug-like compounds via Lipinski's Ro5 filtration. The screening of both the subgroups resulted in 717 (natural) and 2061 (synthetic) compounds, which were further subjected to molecular docking with the Hpse molecular structure. The crystal structure of human Hpse complexed with a tetrasaccharide inhibitor dp4 (PDB ID: 5E9C) [56] provided vital insights into the architecture of the Hpse binding cleft. Hpse is produced as a preproenzyme, and after proteolytic activation, two distinct subunits, including the N-terminal $8 \mathrm{kDa}$ (residues Gln36-Glu109) and C-terminal $50 \mathrm{kDa}$ (residues Lys158-Ile543), produced a mature form of Hpse [4,27,57]. The catalytic site of Hpse is characterized by a narrow channel, with active site residues, Glu225 (proton donor) and Glu343 (proton acceptor) placed in the middle of the channel [58]. The inhibitor binding site is characterized on one side by the heparin binding domain 2 (HBD2; residues 270-280) and on the other side by heparin binding domain 1 (HBD1; residues 158-171) accommodating the terminal iduronic acid of inhibitor dp4 in PDB ID: 5E9C. The model of Hpse built recently by Madia et al. was utilized for our molecular docking studies to devise a putative binding mode for our drug-like compounds with Hpse [27]. This model was built by adding the connecting GS3 $(($ Gly-Ser $) \times 3)$ peptide using MODELER 9.16 software, in which the $8 \mathrm{kDa}$ and $50 \mathrm{kDa}$ subunits are connected, and is, therefore, referred to as the catalytically active form of Hpse. Molecular docking with Hpse resulted in 15 (natural) and 18 (synthetic) compounds demonstrating higher Goldscores and lower Chemscores than the reference inhibitors (Table S1). The above 33 compounds also demonstrated interactions with the key residues of the Hpse binding pocket. Therefore, the acquired 33 compounds were evaluated in physiological conditions by molecular dynamics simulations, and their binding free energies were computed by MM/PBSA calculations (Table S1). The calculated energies were compared with the energy values of the reference inhibitors. REF1 and REF2 displayed BFE values of $-74.612 \mathrm{~kJ} / \mathrm{mol}$ and $-83.519 \mathrm{~kJ} / \mathrm{mol}$, respectively (Table 3 and Table S1). The MM/PBSA calculations revealed better BFE values for two of our identified hits with Hit1 and Hit2 from natural compounds, demonstrating $-104.579 \mathrm{~kJ} / \mathrm{mol}$ and $-83.751 \mathrm{~kJ} / \mathrm{mol}$, respectively (Table 3 and Table S2). The BFE calculations for hits obtained from synthetic compounds revealed Hit1 and Hit2, with considerably better values of $-96.193 \mathrm{~kJ} / \mathrm{mol}$ and $-86.806 \mathrm{~kJ} / \mathrm{mol}$, respectively (Table 3 and Table S2). The BFE scores by $\mathrm{MM} / \mathrm{PBSA}$ enable the entropic distribution of the total $\Delta \mathrm{G}_{\text {bind }}$ energy into identifiable contributions. These individual contributions are characterized by van der Waals, electrostatic, polar solvation and SASA energy. As observed from the BFE distribution analysis, the van der Waals and electrostatic forces provided the maximum driving force for binding of our hits with Hpse. Additionally, the contribution of van der Waals interaction in binding of Hit1 from natural $(-169.280 \pm 18.050 \mathrm{~kJ} / \mathrm{mol})$ as well as synthetic $(-163.420 \pm 16.897 \mathrm{~kJ} / \mathrm{mol})$ sources was observed to be near the contributing range of van der Waals interaction in binding of REF2 $(-173.780 \pm 16.684 \mathrm{~kJ} / \mathrm{mol})$ (Table S2). The entropic distribution of the total BFE suggests that our hits also contribute comparably better in terms of SASA energy and electrostatic energy (Table S2).

The hits achieved from natural and synthetic compounds were further scrutinized for their molecular interactions with Hpse catalytic site residues. Literature reviews on the residues targeting Hpse via hydrogen bonds revealed Glu225, Asn227, Lys231, Gln270, Arg272, Lys274, Glu343, Gly349, Gly350 and Ala388 as vital for Hpse inhibition [27,57-59]. Accordingly, our obtained hits were observed to target Gln270, Asn227, Lys274, Gly349 and Gly350 via hydrogen bonds (Table 4, Figure 5A,B and Figure 6A,B). Further assessment of the literature revealed that the previously reported inhibitors with effective biological activity against Hpse targeted residues Ser163, Glu225, Asn227, Lys231, Gln270, Arg272, Lys274, His296, Tyr298, Glu343, Tyr348, Gly349, Gly350, Gly351, Glu383, Ala388, Gly389 and Tyr391 via hydrophobic and/or van der Waals interactions [23,27,57-59]. Consequently, our hits also demonstrated interactions with the above residues characterized by van der Waals- and $\pi$-mediated bonds (Table 4, Figure 5C,D and Figure 6C,D). As perceived from the hydrogen bond analysis plots, our hits demonstrated a higher number of bonds than the 
reference inhibitors (Figure S3C,D). Moreover, the hits were found to be stable throughout the 50 ns of simulation, as seen from their RMSD (Figure 4A,B) and potential energy plots (Figure S3A,B). The Hpse-hit complexes were also scrutinized at $0 \mathrm{~ns}$ and 50 ns to observe the difference in their interactions with Hpse active site residues (Figure S5). It was perceived that our hits formed higher number of van der Waals interactions at $0 \mathrm{~ns}$, while most of these interactions were lost at the end of $50 \mathrm{~ns}$. Additionally, the superimposed complex structures revealed a slight deviation in the binding pose of Hit1 from the synthetic source at $50 \mathrm{~ns}$. Despite the pose deviancy, it was noticed that Hit1 retained similar interactions with the catalytic site residues of Hpse (Figure S5C). Correspondingly, the alignment of identified hits with Hypo1 indicated that our hits portray the pharmacophoric features reasonably well (Figure S6).

Additionally, to further confirm the toxicity properties of our final hit compounds, the Toxicity Prediction (TOPKAT) module implanted in DS was utilized to evaluate three properties. The TOPKAT module depends on the notion of Quantitative Structure-Toxicity Relationship (QSTR) models and computes toxicity properties which include rodent carcinogenicity, AMES mutagenicity and skin irritancy. According to the U.S. National Toxicology Program (NTP), the compound's rodent carcinogenicity property is evaluated by testing it in both sexes of mouse. The TOPKAT results demonstrated our hits to be non-carcinogenic in both sexes of mouse models. Furthermore, our hits were also observed to be non-AMES mutagenic and non-skin irritant (Table 5). Investigation of oral administration and pharmacokinetic (PK) properties of final hit compounds is essential to avoid their failure in clinical trials. Therefore, the PK properties of our identified hits as well as those of REF inhibitors were calculated and compared by the online tool $p k C S M$ (Table 6) (accessed on 12 May 2021, http:/ / biosig.unimelb.edu.au/pkcsm/) [60]. Given an input molecule, $p k C S M$ predicts PK properties using graph-based signatures. Accordingly, $p k C S M$ predicts various properties encompassing molecular weight, rotatable bonds, water solubility, intestinal absorption, BBB permeability, CYP2D6 inhibitory activity, hERG inhibitory prediction, total clearance and renal OCT2 substrate prediction. The $p k C S M$ results demonstrated that our hits display a moderate level of water solubility, thus confirming that they have good oral bioavailability. Moreover, Caco-2 cell lines are widely employed as an in vitro model in pre-clinical investigations for predicting the likely gastrointestinal permeability of drugs [61]. It was observed that Hit2 from a natural source and Hit1 from a synthetic source displayed better Caco-2 permeability than the REF inhibitors, while Hit1 from a natural source and Hit2 from a synthetic source demonstrated a permeability closer to the acceptable range. A literature survey revealed that molecules with an intestinal absorption (IA) level of $<30 \%$ are classified as being poorly soluble. Intriguingly, all our identified hits displayed IA levels $>30 \%$, similar to that observed for the potent Hpse REF inhibitors. Additionally, the skin permeability property of a given compound of interest is considered for the development of transdermal drug delivery, and it was observed that our hits demonstrated acceptable skin permeability scores $(>-2.5)$. The P-glycoprotein (P-gp) is an extensively studied ATP-binding cassette $(\mathrm{ABC})$ transporter regulating the uptake and efflux of drugs, thereby helping in their absorption [62]. In the present study, our identified hits were observed to be P-gp substrates similar to REF1, except for Hit1 from a synthetic source and REF2. Moreover, all of our hits were predicted to be inhibitors of P-gp. Furthermore, our hit compounds displayed low BBB permeability, thereby limiting the chances of nervous system-related toxicity. The metabolic performance of our hits and REF inhibitors was also assessed by CYP2D6 isoform of cytochrome P450 inhibition. The pkCSM results predicted that our hits were observed to be non-inhibitors of CYP2D6 similar to REF inhibitors, and thus, can be metabolized in the liver. The total clearance (TC) parameter for the excretion of drugs exhibited that Hit 1 from a natural source demonstrates an acceptable TC value similar to REF1, while other hits displayed low TC values similar to that observed for REF2. In addition, our hits were not found to be substrates of renal organic cation transporter 2 (OCT2), which is an essential factor to be considered for the renal clearance of drugs. This illustrated that our hits do not have the potential for adverse interactions 
with co-administered OCT2 inhibitors. Additionally, hERG I is an essential determinant of normal repolarization of cardiac action potential, and its inhibition leads to cardiotoxicity. The $p k C S M$ results predicted that our hits do not inhibit hERG I. Finally, it was perceived that even though REF inhibitors demonstrate good inhibitory activities against Hpse, they do not obey all the Lipinski's Ro5 rules. Both REF1 and REF2 display a molecular weight of more than $500 \mathrm{Da}$ and lipophilicity ( $\log \mathrm{P})$ on the higher level. Compared to REF inhibitors, our hits obeyed all the rules, except for Hit1 from a synthetic source, which displayed LogP slightly greater than the acceptable limit. The above overall properties and the low molecular weights of our hit compounds plays an essential role in their oral absorption. At this stage, note that there are numerous servers for predicting PK properties and the results of different servers are not always the same.

Table 5. Toxicity properties of identified natural and synthetic compound hits generated by TOPKAT.

\begin{tabular}{llccc}
\hline $\begin{array}{c}\text { Hits } \\
\text { (IBS }{ }^{\mathbf{a}} \text { ID) }\end{array}$ & $\begin{array}{c}\text { Mouse Female } \\
\text { Carcinogenicity }\end{array}$ & $\begin{array}{c}\text { Mouse Male } \\
\text { Carcinogenicity }\end{array}$ & $\begin{array}{c}\text { AMES } \\
\text { Mutagenicity }\end{array}$ & $\begin{array}{c}\text { Skin } \\
\text { Irritancy }\end{array}$ \\
\hline Hit1 (STOCK1N-70463) & Non-Carcinogen & Non-Carcinogen & Non-Mutagen & Non-Irritant \\
\hline Hit2 (STOCK1N-48729) & Non-Carcinogen & Non-Carcinogen & Non-Mutagen & Non-Irritant \\
\hline & & Synthetic Compound Hits & & \\
\hline Hit1 (STOCK1S-95244) & Non-Carcinogen & Non-Carcinogen & Non-Mutagen & Non-Irritant \\
\hline Hit2 (STOCK1S-71515) & Non-Carcinogen & Non-Carcinogen & Non-Mutagen & Non-Irritant \\
\hline & ${ }^{a}$ IBS: InterBioScreen; ${ }^{b}$ AMES: Salmonella typhimurium reverse mutation assay. &
\end{tabular}

In addition to the aforementioned analysis, the identified natural and synthetic hits were searched in the PubChem chemistry database (accessed on 15 April 2021, https: / / pubchem.ncbi.nlm.nih.gov/) [63] by entering their SMILES (simplified molecular-input line-entry system) IDs [64] to check if our hits have been evaluated in the literature, against Hpse. From the PubChem analysis, it was observed that our hits have not been assessed against Hpse before, and hence, can be considered as valuable therapeutics against Hpsemediated ailments. Moreover, from the IUPAC name of our hits, it can be perceived that Hit2 from both synthetic and natural compounds represent compounds from benzamide origin (Table 7) and similar molecules of this source have been explored and reported before by $\mathrm{Xu}$ et al. [31]. On the other hand, Hit1 from both natural and synthetic compounds indicate molecules of acetamide and sulfonamide origin, respectively (Table 7). The small molecules from the aforementioned origins have not been reported in the literature yet. Overall, we anticipate that our hits may be effective drug candidates as potent therapeutics and can be recommended for further evaluation against Hpse.

The laboratory in vitro research necessitates chemicals and other techniques, which is a time-consuming and tedious process [65]. Therefore, we accessed the experiment-free prediction method for assessing the inhibitory behavior of our hit compounds. Deep learning models have progressed recently to predict the inhibitory activity of the compounds. PaccMann (accessed on 12 May 2021, https://ibm.biz/paccmann-aas) is one such web-based drug sensitivity platform designed to utilize multimodal attention-based neural networks [66]. Moreover, PaccMann is an effective validation toolbox used for drug repurposing approaches and has an $\mathrm{R}^{2}$ value of 0.86 along with an RMSE (root mean square error) value of 0.89 , highlighting the strong correlations between the resultant data generated by the server and the experimentally estimated values. Accordingly, the SMILES IDs of our hit compounds and REF inhibitors were supplied to the platform as an input and the sensitivity against cancer cell lines was predicted in terms of their $\mathrm{IC}_{50}$ values. The $\mathrm{IC}_{50}$ values were estimated for the ovarian (A2780), lung (A549) and breast (MCF-7) cancer cell lines, as Hpse is overexpressed in the aforementioned particular cancers. Additionally, the $\mathrm{IC}_{50}$ values were also predicted for Ewing's sarcoma (SK-ES-1), multiple myeloma 
(MM1S) and hepatocellular carcinoma (HepG2) cell lines, owing to Hpse dysregulation in the cancer subtypes. The prediction of $\mathrm{IC}_{50}$ values for all hits was observed to be low or in the similar range as that for REF inhibitors in all cancer cell lines, except for Hit2 from a synthetic source, which was observed to predict higher $\mathrm{IC}_{50}$ values (Table S3). A similar study was also recently performed by Thirunavukkarasu et al. [67], who utilized the PaccMann server to successfully predict the anticancer sensitivity on 77 lung cancer cell lines.

Table 6. In silico assessment of pharmacokinetic (PK) properties for reference (REF) inhibitors and identified hits generated by $p k C S M$.

\begin{tabular}{|c|c|c|c|c|c|c|c|}
\hline \multirow{2}{*}{$\begin{array}{c}\text { PK } \\
\text { Properties }\end{array}$} & \multicolumn{2}{|c|}{$\begin{array}{c}\text { Natural Compound } \\
\text { Hits }\end{array}$} & \multicolumn{2}{|c|}{$\begin{array}{c}\text { Synthetic Compound } \\
\text { Hits }\end{array}$} & \multicolumn{2}{|c|}{$\begin{array}{l}\text { Reference } \\
\text { Inhibitors }\end{array}$} & \multirow[b]{2}{*}{ Cut-Off } \\
\hline & $\begin{array}{c}\text { Hit1 } \\
\text { (STOCK1N- } \\
\text { 70463) } \\
\end{array}$ & $\begin{array}{c}\text { Hit2 } \\
\text { (STOCK1N- } \\
48729) \\
\end{array}$ & $\begin{array}{c}\text { Hit1 } \\
\text { (STOCK1S- } \\
\text { 95244) } \\
\end{array}$ & $\begin{array}{c}\text { Hit2 } \\
\text { (STOCK1S- } \\
71515) \\
\end{array}$ & REF1 & REF2 & \\
\hline $\begin{array}{l}\text { Molecular } \\
\text { weight }\end{array}$ & 447.48 & 474.56 & 489.58 & 489.35 & 500.60 & 598.51 & $\leq 500 \mathrm{Da}$ \\
\hline $\log \mathrm{P}$ & 4.57 & 4.33 & 5.30 & 4.53 & 7.65 & 6.47 & $<5$ \\
\hline Rotatable Bonds & 9 & 7 & 7 & 9 & 4 & 8 & $<10$ \\
\hline HBA & 6 & 4 & 8 & 5 & 3 & 7 & $\leq 10$ \\
\hline HBD & 1 & 2 & 2 & 3 & 4 & 4 & $\begin{array}{c}\leq 5 \\
<-10\end{array}$ \\
\hline Water solubility & -5.585 & -5.078 & -3.182 & -4.998 & -2.892 & -2.905 & $\begin{array}{c}\text { insoluble to } \\
<0 \text { highly } \\
\text { soluble }\end{array}$ \\
\hline $\begin{array}{c}\text { Caco-2 } \\
\text { permeability }\end{array}$ & 0.585 & 1.169 & 1.101 & 0.564 & 0.754 & -0.526 & $>0.90$ \\
\hline IA (human) & 94.26 & 100 & 97.45 & 82.54 & 100 & 64.80 & $>30$ \\
\hline $\begin{array}{c}\text { Skin } \\
\text { permeability }\end{array}$ & -2.688 & -2.802 & -2.735 & -2.752 & -2.735 & -2.735 & $>-2.5$ \\
\hline P-gp substrate & Yes & Yes & No & Yes & Yes & No & No \\
\hline P-gp I inhibitor & Yes & Yes & Yes & Yes & No & No & No \\
\hline BBB permeability & -0.862 & -0.854 & -0.633 & -1.136 & -0.941 & -2.352 & $\begin{array}{l}>0.3 \text { high to } \\
<-1 \text { poor }\end{array}$ \\
\hline $\begin{array}{l}\text { CYP2D6 } \\
\text { inhibitor }\end{array}$ & No & No & No & No & No & No & No \\
\hline hERG I inhibitor & No & No & No & No & Yes & No & No \\
\hline Total clearance & 0.544 & 0.152 & -0.023 & -0.124 & 0.813 & -0.171 & $\begin{array}{l}<0.3 \text { low to } \\
>0.7 \text { high }\end{array}$ \\
\hline $\begin{array}{l}\text { Renal OCT2 } \\
\text { substrate }\end{array}$ & No & No & No & No & Yes & No & No \\
\hline
\end{tabular}

Abbreviations-HBA: Hydrogen Bond Acceptor, HBD: Hydrogen Bond Donor, IA: Intestinal Absorption, P-gp: P-glycoprotein, BBB: Blood-Brain Barrier, hERG: human ether-a-go-go-related gene, OCT2: Organic Cation Transporter 2. 
Table 7. Molecular structures and IUPAC names of identified hits from InterBioScreen database.

\begin{tabular}{|c|c|c|}
\hline Compound Name & IUPAC Name & Molecular Structure \\
\hline \multicolumn{3}{|c|}{ Natural Compound Hits } \\
\hline Hit1 & $\begin{array}{l}\mathrm{N}-(4-(\text { furan-2-yl)butan-2-yl)-2-((3-(4- } \\
\text { methoxyphenyl)-4-oxo-4H-chromen-7- } \\
\text { yl)oxy)acetamide }\end{array}$ & \\
\hline Hit2 & $\begin{array}{l}\text { (S)-4-(8-methoxy-11b-methyl-1,3- } \\
\text { dioxo-5,6-dihydro-1H-imidazo[1 } \\
\text { ',5':1,2]pyrido[3,4-b]indol- } \\
\text { 2(3H,11H,11bH)-yl)-N- } \\
\text { pentylbenzamide }\end{array}$ & \\
\hline \multicolumn{3}{|c|}{ Synthetic Compound Hits } \\
\hline Hit1 & $\begin{array}{l}\text { 4-((4-(4-methoxyphenyl)phthalazin-1- } \\
\text { yl)amino)-N-(thiazol-2- } \\
\text { yl)benzenesulfonamide }\end{array}$ & \\
\hline Hit2 & $\begin{array}{c}\text { (E)-N-(1-(5-(2,5-dichlorophenyl)furan- } \\
\text { 2-yl)-3-((3-hydroxypropyl)amino)-3- } \\
\text { oxoprop-1-en-2-yl)-4- } \\
\text { methoxybenzamide }\end{array}$ & \\
\hline
\end{tabular}

\section{Materials and Methods}

\subsection{Dataset Preparation and Pharmacophore Model Generation}

A dataset of four well-known Hpse inhibitors, as reported in the literature [1], composed of distinct scaffolds and different maximal inhibitory concentration $\left(\mathrm{IC}_{50}\right)$ values, was chosen as the training set for the generation of pharmacophore model. These four inhibitors consisted of an acetic acid $\left(\mathrm{IC}_{50}=0.2 \mu \mathrm{M}\right)$ [33], benzamide $\left(\mathrm{IC}_{50}=0.29 \mu \mathrm{M}\right)$ [31], urea $\left(\mathrm{IC}_{50}=0.075 \mu \mathrm{M}\right)$ [30] and symmetrical benzazolyl $\left(\mathrm{IC}_{50}=0.18 \mu \mathrm{M}\right)$ [57] derivatives endowed with Hpse inhibitory activity. Accordingly, the 3D structures of the chosen compounds were downloaded from BindingDB [68], manually checked and energy minimized employing the Minimize Ligands module in Discovery Studio (DS) v.18 (Accelrys, San Diego, CA, USA). Prior to model generation, the Feature Mapping protocol in DS was employed for identifying the common chemical features in the training set compounds. The features predicted from the above-mentioned step were used as inputs for the generation of a model using the Common Feature Pharmacophore Generation tool of DS. This ligand-based pharmacophore approach utilizes the HipHop algorithm to extract features common to a set of limited active molecules [49]. The BEST/Flexible conformation generation, along with an energy threshold of $20 \mathrm{kcal} / \mathrm{mol}$ and interfeature distance of $2.97 \AA$, was used to produce a maximum of 255 conformations. A total of 10 hypotheses are generated with parameters, including the comprised features, hypothesis rank, direct hit, partial hit and maximum fit values.

\subsection{Validation of the Generated Model}

Pharmacophore validation is a crucial step in assessing the pharmacophore robustness for retrieving active compounds from a given dataset. The best pharmacophore hypothesis generated by HipHop was validated by the Güner-Henry (GH) scoring method, also known as the decoy set method [69]. The GH validation was instigated by subjecting the generated pharmacophore model to an external dataset (D) of 100 compounds with four active (A) molecules used in pharmacophore generation. The selected pharmacophore hypothesis was used as a 3D query employing Ligand Pharmacophore Mapping protocol of DS for acquiring the goodness of fit (GF) score in the range of 0 (null model) and 1 (ideal model) [55]. The equation mentioned below was utilized for calculating the GF score value, where Ht 
signifies the total number of hits retrieved by the pharmacophore model and Ha denotes the active molecules retrieved in $\mathrm{Ht}$ :

$$
G F=\left(\frac{H a}{4 H t \mathrm{~A}}\right)(3 \mathrm{~A}+H t) \times\left\{1-\frac{H t-H a}{\mathrm{D}-\mathrm{A}}\right\}
$$

\subsection{Drug-Like Database Generation and Virtual Screening of InterBioScreen Database}

The virtual screening strategy in this study used the validated pharmacophore as a query to search the InterBioScreen (IBS) database composed of natural compounds $(69,034)$ as well as synthetic compounds $(195,469)$. Prior to screening, the compounds were filtered for their drug-like attributes on the basis of Lipinski's Rule of Five (Ro5) and Veber's rule by employing the Filter by Lipinski and Veber Rules module of DS. Ro5 signifies that the potential drug-like compound exhibits a molecular weight of $500 \mathrm{Da}$, an octanol/water partition coefficient $(\log \mathrm{P})$ of less than 5 , less than 5 hydrogen bond donors and 10 hydrogen bond acceptors [70]. Additionally, Veber's rule of less than 10 rotatable bonds was applied to acquire drug-like compounds for further analysis [71]. The validated pharmacophore model was subsequently used to screen these drug-like databases by engaging the Ligand Pharmacophore Mapping protocol of DS using the FAST/Flexible fitting method. The mapped drug-like compounds were consequently chosen for molecular docking with Hpse.

\subsection{Molecular Docking of Screened Drug-Like Compounds with Hpse}

Docking studies were initiated by adopting the model of human GS3 Hpse, previously developed by Madia et al. from the PDB ID: 5E9C (resolution: $1.73 \AA$ ) (accessed on 24 November 2020, www.rcsb.org) [56], where Hpse is complexed with heparin tetrasaccharide inhibitor dp4 [27]. The mature human Hpse (UniProtKB ID: Q9Y251) is a heterodimer structure encompassing two chains-N-terminal $8 \mathrm{kDa}$ (residues Gln36-Glu109) and C-terminal $50 \mathrm{kDa}$ (residues Lys158-Ile543)—noncovalently assembled into a $(\beta / \alpha)_{8}$ TIM barrel fold [57]. The developed model of Hpse was built in a way that the $8 \mathrm{kDa}$ and $50 \mathrm{kDa}$ chains are connected by linker peptide GS3 $(($ Gly-Ser $) \times 3)$ and represents the catalytically active form of human Hpse. The binding site in the Hpse model was defined as a sphere of $16 \AA$ by using the Define and Edit Binding Site tool of DS with X, Y and $\mathrm{Z}$ co-ordinates of $-22.86,13.98$ and 59.98, respectively. Prior to the docking process, the Genetic Optimization for Ligand Docking (GOLD v5.2.2) [72] protocol was validated by re-docking the co-crystallized benzazolyl inhibitor of the aforementioned Hpse model. The drug-like natural and synthetic compounds obtained from virtual screening of IBS database were minimized and prepared by employing the Minimize Ligands DS protocol. Consequently, the compounds acquired were docked with the Hpse model by allowing for generation of 10 conformers per ligand. The obtained conformations were clustered to achieve the largest cluster, from which the compounds were evaluated on the basis of two scoring criterions [73]—Goldscore (high) and Chemscore (low) [74] —as well as the molecular interactions with the Hpse catalytic site residues (Asp62, Asn64, Thr97, Glu225, Asn227, Lys231, Gln270, Arg272, Glu343, Gly349, Gly350, Ala388 and Tyr391).

\subsection{Molecular Dynamics Simulation of Identified Natural and Synthetic Compounds}

Molecular dynamics (MD) simulations are applied to understand the protein-ligand interactions at the atomic level in order to scrutinize their conformational flexibility and structural stability under physiological conditions $[75,76]$. The complexes obtained from the process of molecular docking were subjected to MD simulations in GROningen MAchine for Chemical Simulations (GROMACS v2018) [77] with the docked structures of compounds with Hpse as initial coordinates. The protein and the compounds were parametrized by CHARMm27 [78] and SwissParam [79] fast force field generation tools, respectively. All simulation systems were immersed in a dodecahedron water box of TIP3P solvent model and neutralized by the addition of $16 \mathrm{Cl}^{-}$ions. Prior to system equilibration, energy minimization of each simulation system was performed by the steepest descent algorithm in order to avoid steric clashes [80]. Systems were further subjected to two-stage equilibra- 
tion process composed of NVT (constant number of particles, volume and temperature) and NPT (constant number of particles, pressure and temperature) for $500 \mathrm{ps}$ each. The NVT ensemble uses a V-rescale thermostat [81] to equilibrate the system temperature at $300 \mathrm{~K}$, while the NPT ensemble uses the Parrinello-Rahman barostat [82] to maintain the system pressure at 1.0 bar. Systems equilibrated by the above steps were subjected to MD simulation run of $50 \mathrm{~ns}$ each under periodic boundary conditions. Long-range electrostatic interactions were calculated with Particle Mesh Ewald (PME) [83] approach with a cutoff radius of $10 \AA$ and the LINCS algorithm [84] restrained the bond lengths of heavy atoms. The MD outcomes were further visualized and scrutinized in DS and visual molecular dynamics (VMD) [85].

\subsection{Binding Free Energy Calculations}

Calculation of the binding affinity of small molecule inhibitors with their target proteins represents a quintessential role to prioritize compounds before their experimental evaluation [86]. To evaluate the binding affinities of our compounds with Hpse, the Molecular Mechanics Poisson-Boltzmann Surface Area (MM/PBSA) in GROMACS was utilized by implementing the ' $g \_m m p b s a$ ' tool [87]. For this purpose, 50 snapshots were selected evenly for the entire simulation run of $50 \mathrm{~ns}$ and the binding free energy $\Delta G_{\text {bind }}$ was computed as:

$$
\Delta G_{\text {bind }}=G_{\text {complex }}\left(G_{\text {protein }}+G_{\text {ligand }}\right)
$$

\section{Conclusions}

A ligand-based common-feature pharmacophore model exploiting the features shared by a set of active inhibitors revealed essential criteria required for Heparanase inhibition. The model composed of six pharmacophoric features was validated and subsequently used as a query to screen drug-like compounds from the InterBioScreen database. A total of 2778 drug-like compounds acquired from pharmacophore mapping were assessed by molecular docking with Heparanase to gain insight on their binding. The 33 obtained compounds from docking analysis exhibited higher docking scores than the reference inhibitors and were able to inhibit Heparanase heterodimer by interacting with key active site residues. The acquired compounds were further evaluated in physiological conditions via molecular dynamics simulations and their binding affinities with Heparanase were computed by MM/PBSA calculations. Analysis of binding affinity revealed two hit compounds each from natural and synthetic databases displaying higher binding affinity than reference inhibitors for Heparanase inhibition. Additionally, the intermolecular interaction analysis revealed that the selected hits interact with key catalytic residues via hydrogen bonds, thus providing support in their selection as hit molecules. Furthermore, compounds of acetamide and sulfonamide scaffolds have not been previously reported as Hpse inhibitors. Therefore, compounds comprising aforementioned scaffolds can be considered as a novel source for future identification of Heparanase inhibitors. We anticipate that the identified scaffolds of hit compounds can be considered for drug optimization against Heparanase in the future. 
Supplementary Materials: The following are available online at https://www.mdpi.com/article/10 $.3390 /$ ijms22105311/s1, Figure S1. Chemical structures of carbohydrate-based, nucleic acid-based and small molecule Heparanase inhibitors. Figure S2. Overlay of the docked pose (orange) of bound ligand with its GS3 Heparanase model conformation (mauve). Figure S3. Molecular dynamics simulation analysis plots of Heparanase with the reference (REF) and Hits displaying (A and B) potential energy and ( $C$ and $D$ ) hydrogen bonds. The left (A and $C$ ) and right (B and $D)$ columns represent analysis for natural and synthetic compound hits, respectively. Figure S4. The 3D and 2D intermolecular interactions of reference (REF) compounds (REF1: A and C; REF2: B and D) with the active site residues of Heparanase. Figure S5. The superimposed complex structures and 2D intermolecular interactions of natural and synthetic compound hits with the catalytic residues of Heparanase at $0 \mathrm{~ns}$ and $50 \mathrm{~ns}$. Figure S6. Alignment of identified hits with the pharmacophoric features of Hypo1. Subsections A and B represent Hit1 and Hit2 from natural compounds, respectively, whereas subsections $\mathrm{C}$ and D denote Hit1 and Hit2 from synthetic compounds of InterBioScreen database, respectively. Table S1. The docking and binding free energy scores of drug-like natural and synthetic compounds from the InterBioScreen (IBS) database with Heparanase. Table S2. The entropic distribution of the total binding free energy scores for reference (REF) inhibitors and selected potential hits from the InterBioScreen (IBS) database against Heparanase. Table S3. Assessment of anti-cancer drug sensitivity prediction for reference (REF) inhibitors and identified hits generated by PaccMann.

Author Contributions: Conceptualization, S.P. and V.K.; methodology, S.P. and V.K.; software, S.P. and V.K.; validation, S.P. and V.K.; formal analysis, S.P., V.K. and D.; investigation, S.P. and V.K.; resources, K.W.L.; data curation, S.P., V.K. and D.; writing-original draft preparation, S.P. and V.K.; writing-review and editing, S.P. and V.K.; visualization, S.P. and V.K.; supervision, K.W.L.; project administration, K.W.L. and J.C.H.; funding acquisition, K.W.L. and J.C.H. All authors have read and agreed to the published version of the manuscript.

Funding: This research was supported by the Basic Science Research Program through the National Research Foundation of Korea (NRF) funded by the Ministry of Education (2020R1A6A1A03044344). This research was supported by the Bio \& Medical Technology Development Program of the National Research Foundation (NRF) \& funded by the Korean government (MSIT) (No. NRF-2018M3A9A7057263).

Institutional Review Board Statement: Not applicable.

Informed Consent Statement: Not applicable.

Data Availability Statement: Data are contained within the article.

Conflicts of Interest: The authors declare no conflict of interest.

\section{References}

1. Mohan, C.D.; Hari, S.; Preetham, H.D.; Rangappa, S.; Barash, U.; Ilan, N.; Nayak, S.C.; Gupta, V.K.; Basappa; Vlodavsky, I.; et al. Targeting Heparanase in Cancer: Inhibition by Synthetic, Chemically Modified, and Natural Compounds. iScience 2019, 15, 360-390. [CrossRef] [PubMed]

2. Baburajeev, C.P.; Mohan, C.D.; Rangappa, S.; Mason, D.J.; Fuchs, J.E.; Bender, A.; Barash, U.; Vlodavsky, I.; Basappa; Rangappa, K.S. Identification of Novel Class of Triazolo-Thiadiazoles as Potent Inhibitors of Human Heparanase and their Anticancer Activity. BMC Cancer 2017, 17, 1-14. [CrossRef] [PubMed]

3. Vlodavsky, I.; Singh, P.; Boyango, I.; Gutter-Kapon, L.; Elkin, M.; Sanderson, R.D.; Ilan, N. Heparanase: From basic research to therapeutic applications in cancer and inflammation. Drug Resist. Updat. 2016, 29, 54-75. [CrossRef] [PubMed]

4. Coombe, D.R.; Gandhi, N.S. Heparanase: A Challenging Cancer Drug Target. Front. Oncol. 2019, 9. [CrossRef]

5. Ramani, V.C.; Yang, Y.; Ren, Y.; Nan, L.; Sanderson, R.D. Heparanase plays a dual role in driving hepatocyte growth factor (HGF) signaling by enhancing HGF expression and activity. J. Biol. Chem. 2011, 286, 6490-6499. [CrossRef]

6. Purushothaman, A.; Chen, L.; Yang, Y.; Sanderson, R.D. Heparanase stimulation of protease expression implicates it as a master regulator of the aggressive tumor phenotype in myeloma. J. Biol. Chem. 2008, 283, 32628-32636. [CrossRef]

7. Zetser, A.; Bashenko, Y.; Edovitsky, E.; Levy-Adam, F.; Vlodavsky, I.; Ilan, N. Heparanase induces vascular endothelial growth factor expression: Correlation with p38 phosphorylation levels and Src activation. Cancer Res. 2006, 66, 1455-1463. [CrossRef]

8. Ilan, N.; Elkin, M.; Vlodavsky, I. Regulation, function and clinical significance of heparanase in cancer metastasis and angiogenesis. Int. J. Biochem. Cell Biol. 2006, 38, 2018-2039. [CrossRef]

9. Cassinelli, G.; Favini, E.; Bo, L.D.; Tortoreto, M.; De Maglie, M.; Dagrada, G.; Pilotti, S.; Zunino, F.; Zaffaroni, N.; Lanzi, C. Antitumor efficacy of the heparan sulfate mimic roneparstat (SST0001) against sarcoma models involves multi-target inhibition of receptor tyrosine kinases. Oncotarget 2016, 7, 47848-47863. [CrossRef] 
10. Vlodavsky, I.; Beckhove, P.; Lerner, I.; Pisano, C.; Meirovitz, A.; Ilan, N.; Elkin, M. Significance of heparanase in cancer and inflammation. Cancer Microenviron. 2012, 5, 115-132. [CrossRef]

11. Pisano, C.; Vlodavsky, I.; Ilan, N.; Zunino, F. The potential of heparanase as a therapeutic target in cancer. Biochem. Pharmacol. 2014, 89, 12-19. [CrossRef]

12. Rivara, S.; Milazzo, F.M.; Giannini, G. Heparanase: A rainbow pharmacological target associated to multiple pathologies including rare diseases. Future Med. Chem. 2016, 8, 647-680. [CrossRef]

13. Khasraw, M.; Pavlakis, N.; McCowatt, S.; Underhill, C.; Begbie, S.; de Souza, P.; Boyce, A.; Parnis, F.; Lim, V.; Harvie, R.; et al. Multicentre phase I/II study of PI-88, a heparanase inhibitor in combination with docetaxel in patients with metastatic castrateresistant prostate cancer. Ann. Oncol. 2009, 21, 1302-1307. [CrossRef]

14. A Phase III PI-88 in the Adjuvant Treatment of Subjects with Hepatitis Virus Related HCC After Surgical Resection-Tabular ViewClinicalTrials.gov. Available online: https:/ / clinicaltrials.gov/ct2/show/record/NCT01402908 (accessed on 27 February 2021).

15. SST0001 (Roneparstat) in Advanced Multiple Myeloma-Full Text View-ClinicalTrials.gov. Available online: https: / / clinicaltrials.gov / ct2/show / study / NCT01764880 (accessed on 27 February 2021).

16. Ritchie, J.P.; Ramani, V.C.; Ren, Y.; Naggi, A.; Torri, G.; Casu, B.; Penco, S.; Pisano, C.; Carminati, P.; Tortoreto, M.; et al. SST0001, a chemically modified heparin, inhibits myeloma growth and angiogenesis via disruption of the heparanase/syndecan-1 axis. Clin. Cancer Res. 2011, 17, 1382-1393. [CrossRef]

17. Study of the Safety and Tolerability of IV Infused PG545 in Patients with Advanced Solid Tumours-Full Text ViewClinicalTrials.gov. Available online: https:/ / clinicaltrials.gov/ct2/show/NCT02042781 (accessed on 27 February 2021).

18. Dredge, K.; Hammond, E.; Handley, P.; Gonda, T.J.; Smith, M.T.; Vincent, C.; Brandt, R.; Ferro, V.; Bytheway, I. PG545, a dual heparanase and angiogenesis inhibitor, induces potent anti-tumour and anti-metastatic efficacy in preclinical models. Br. J. Cancer 2011, 104, 635-642. [CrossRef]

19. Zhou, H.; Roy, S.; Cochran, E.; Zouaoui, R.; Chu, C.L.; Duffner, J.; Zhao, G.; Smith, S.; Galcheva-Gargova, Z.; Karlgren, J.; et al. M402, a novel Heparan sulfate mimetic, targets multiple pathways implicated in tumor progression and metastasis. PLoS ONE 2011, 6, e21106. [CrossRef]

20. M402 in Combination with Nab-Paclitaxel and Gemcitabine in Pancreatic Cancer-Full Text View-ClinicalTrials.gov. Available online: https: / / clinicaltrials.gov/ct2/ show / NCT01621243 (accessed on 27 February 2021).

21. Zhao, H.; Liu, H.; Chen, Y.; Xin, X.; Li, J.; Hou, Y.; Zhang, Z.; Zhang, X.; Xie, C.; Geng, M.; et al. Oligomannurarate sulfate, a novel heparanase inhibitor simultaneously targeting basic fibroblast growth factor, combats tumor angiogenesis and metastasis. Cancer Res. 2006, 66, 8779-8787. [CrossRef]

22. Parish, C.R.; Coombe, D.R.; Jakobsen, K.B.; Bennett, F.A.; Underwood, P.A. Evidence that sulphated polysaccharides inhibit tumour metastasis by blocking tumour-cell-derived heparanases. Int. J. Cancer 1987, 40, 511-518. [CrossRef]

23. Ishida, K.; Hirai, G.; Murakami, K.; Teruya, T.; Simizu, S.; Sodeoka, M.; Osada, H. Structure-based design of a selective heparanase inhibitor as an antimetastatic agent. Mol. Cancer Ther. 2004, 3, 1069-1077.

24. Hamaguchi, T.; Sudo, T.; Osada, H. RK-682, a potent inhibitor of tyrosine phosphatase, arrested the mammalian cell cycle progression at G1phase. FEBS Lett. 1995, 372, 54-58. [CrossRef]

25. Heyman, B.; Yang, Y. Mechanisms of heparanase inhibitors in cancer therapy. Exp. Hematol. 2016, 44, 1002-1012. [CrossRef]

26. Echart, C.; Distaso, M.; Baraz, L.; Vlodavsky, I.; Iacobelli, M. Defibrotide downregulates Heparanase expression in multiple myeloma, renal adenocarcinoma and breast cancer cell lines. Cancer Res. 2008, 68, 1180.

27. Madia, V.N.; Messore, A.; Pescatori, L.; Saccoliti, F.; Tudino, V.; De Leo, A.; Bortolami, M.; Scipione, L.; Costi, R.; Rivara, S.; et al. Novel Benzazole Derivatives Endowed with Potent Antiheparanase Activity. J. Med. Chem. 2018, 61, 6918-6936. [CrossRef] [PubMed]

28. De Clercq, E. Suramin: A potent inhibitor of the reverse transcriptase of RNA tumor viruses. Cancer Lett. 1979, 8, 9-22. [CrossRef]

29. Nakajima, M.; DeChavigny, A.; Johnson, C.E.; Hamada, J.I.; Stein, C.A.; Nicolson, G.L. Suramin: A potent inhibitor of melanoma heparanase and invasion. J. Biol. Chem. 1991, 266, 9661-9666. [CrossRef]

30. Pan, W.; Miao, H.Q.; Xu, Y.J.; Navarro, E.C.; Tonra, J.R.; Corcoran, E.; Lahiji, A.; Kussie, P.; Kiselyov, A.S.; Wong, W.C.; et al. 1-[4-(1H-Benzoimidazol-2-yl)-phenyl]-3-[4-(1H-benzoimidazol-2-yl)-phenyl] -urea derivatives as small molecule heparanase inhibitors. Bioorg. Med. Chem. Lett. 2006, 16, 409-412. [CrossRef] [PubMed]

31. Xu, Y.J.; Miao, H.Q.; Pan, W.; Navarro, E.C.; Tonra, J.R.; Mitelman, S.; Camara, M.M.; Deevi, D.S.; Kiselyov, A.S.; Kussie, P.; et al. $\mathrm{N}-(4-\{[4-(1 \mathrm{H}-$ Benzoimidazol-2-yl)-arylamino]-methyl $\}$-phenyl)-benzamide derivatives as small molecule heparanase inhibitors. Bioorg. Med. Chem. Lett. 2006, 16, 404-408. [CrossRef]

32. Courtney, S.M.; Hay, P.A.; Buck, R.T.; Colville, C.S.; Porter, D.W.; Scopes, D.I.C.; Pollard, F.C.; Page, M.J.; Bennett, J.M.; Hircock, M.L.; et al. 2,3-Dihydro-1,3-dioxo-1H-isoindole-5-carboxylic acid derivatives: A novel class of small molecule heparanase inhibitors. Bioorg. Med. Chem. Lett. 2004, 14, 3269-3273. [CrossRef]

33. Courtney, S.M.; Hay, P.A.; Buck, R.T.; Colville, C.S.; Phillips, D.J.; Scopes, D.I.C.; Pollard, F.C.; Page, M.J.; Bennett, J.M.; Hircock, M.L.; et al. Furanyl-1,3-thiazol-2-yl and benzoxazol-5-yl acetic acid derivatives: Novel classes of heparanase inhibitor. Bioorganic Med. Chem. Lett. 2005, 15, 2295-2299. [CrossRef]

34. Mohan, C.D.; Bharathkumar, H.; Bulusu, K.C.; Pandey, V.; Rangappa, S.; Fuchs, J.E.; Shanmugam, M.K.; Dai, X.; Li, F.; Deivasigamani, A.; et al. Development of a novel azaspirane that targets the Janus Kinase-signal transducer and activator of transcription (STAT) pathway in hepatocellular carcinoma in vitro and in vivo. J. Biol. Chem. 2014, 289, 34296-34307. [CrossRef] 
35. Bharathkumar, H.; Mohan, C.D.; Rangappa, S.; Kang, T.; Keerthy, H.K.; Fuchs, J.E.; Kwon, N.H.; Bender, A.; Kim, S.; Basappa; et al. Screening of quinoline, 1,3-benzoxazine, and 1,3-oxazine-based small molecules against isolated methionyl-tRNA synthetase and A549 and HCT116 cancer cells including an in silico binding mode analysis. Org. Biomol. Chem. 2015, 13, 9381-9387. [CrossRef]

36. Srinivas, V.; Mohan, C.D.; Baburajeev, C.P.; Rangappa, S.; Jagadish, S.; Fuchs, J.E.; Sukhorukov, A.Y.; Chandra; Mason, D.J.; Sharath-Kumar, K.S.; et al. Synthesis and characterization of novel oxazines and demonstration that they specifically target cyclooxygenase 2. Bioorg. Med. Chem. Lett. 2015, 25, 2931-2936. [CrossRef]

37. Nirvanappa, A.C.; Mohan, C.D.; Rangappa, S.; Ananda, H.; Sukhorukov, A.Y.; Shanmugam, M.K.; Sundaram, M.S.; Nayaka, S.C.; Girish, K.S.; Chinnathambi, A.; et al. Novel Synthetic Oxazines Target NF-kB in Colon Cancer In Vitro and Inflammatory Bowel Disease In Vivo. PLoS ONE 2016, 11, e0163209. [CrossRef]

38. Sulaiman, N.B.; Mohan, C.D.; Basappa, B.; Pandey, V.; Rangappa, S.; Bharathkumar, H.; Kumar, A.P.; Lobie, P.E.; Rangappa, K.S. An azaspirane derivative suppresses growth and induces apoptosis of ER-positive and ER-negative breast cancer cells through the modulation of JAK2/STAT3 signaling pathway. Int. J. Oncol. 2016, 49, 1221-1229. [CrossRef]

39. Basappa; Murugan, S.; Kavitha, C.V.; Purushothaman, A.; Nevin, K.G.; Sugahara, K.; Rangappa, K.S. A small oxazine compound as an anti-tumor agent: A novel pyranoside mimetic that binds to VEGF, HB-EGF, and TNF- $\alpha$. Cancer Lett. 2010, 297, $231-243$. [CrossRef]

40. Uzma, F.; Mohan, C.D.; Hashem, A.; Konappa, N.M.; Rangappa, S.; Kamath, P.V.; Singh, B.P.; Mudili, V.; Gupta, V.K.; Siddaiah, C.N.; et al. Endophytic fungi-alternative sources of cytotoxic compounds: A review. Front. Pharmacol. 2018, 9, 309. [CrossRef]

41. Gozalbes, R.; Mosulén, S.; Ortí, L.; Rodríguez-Díaz, J.; Carbajo, R.J.; Melnyk, P.; Pineda-Lucena, A. Hit identification of novel heparanase inhibitors by structure- and ligand-based approaches. Bioorg. Med. Chem. 2013, 21, 1944-1951. [CrossRef]

42. Gozalbes, R.; Mosulén, S.; Carbajo, R.J.; Pineda-Lucena, A. Development and NMR validation of minimal pharmacophore hypotheses for the generation of fragment libraries enriched in heparanase inhibitors. J. Comput. Mol. Des. 2009, 23, 555-569. [CrossRef]

43. Dai, X.; Yan, J.; Fu, X.; Pan, Q.; Sun, D.; Xu, Y.; Wang, J.; Nie, L.; Tong, L.; Shen, A.; et al. Aspirin inhibits cancer metastasis and angiogenesis via targeting heparanase. Clin. Cancer Res. 2017, 23, 6267-6279. [CrossRef]

44. Parate, S.; Kumar, V.; Hong, J.C.; Lee, K.W. Identification of Flavonoids as Putative ROS-1 Kinase Inhibitors Using Pharmacophore Modeling for NSCLC Therapeutics. Molecules 2021, 26, 2114. [CrossRef]

45. Parate, S.; Kumar, V.; Lee, G.; Rampogu, S.; Hong, J.C.; Lee, K.W. Marine-Derived Natural Products as ATP-Competitive mTOR Kinase Inhibitors for Cancer Therapeutics. Pharmaceuticals 2021, 14, 282. [CrossRef]

46. Ferreira, L.G.; Dos Santos, R.N.; Oliva, G.; Andricopulo, A.D. Molecular docking and structure-based drug design strategies. Molecules 2015, 20, 13384-13421. [CrossRef]

47. Jayatilleke, K.M.; Hulett, M.D. Heparanase and the hallmarks of cancer. J. Transl. Med. 2020, 18, 1-25. [CrossRef]

48. Vlodavsky, I.; Ilan, N.; Naggi, A.; Casu, B. Heparanase: Structure, Biological Functions, and Inhibition by Heparin-Derived Mimetics of Heparan Sulfate. Curr. Pharm. Des. 2007, 13, 2057-2073. [CrossRef] [PubMed]

49. Kurogi, Y.; Guner, O. Pharmacophore Modeling and Three-dimensional Database Searching for Drug Design Using Catalyst. Curr. Med. Chem. 2012, 8, 1035-1055. [CrossRef] [PubMed]

50. Patel, Y.; Gillet, V.J.; Bravi, G.; Leach, A.R. A comparison of the pharmacophore identification programs: Catalyst, DISCO and GASP. J. Comput. Mol. Des. 2002, 16, 653-681. [CrossRef]

51. Ataei, S.; Yilmaz, S.; Ertan-Bolelli, T.; Yildiz, I. Generated 3D-Common Feature Hypotheses Using the HipHop Method for Developing New Topoisomerase i Inhibitors. Arch. Pharm. 2015, 348, 498-507. [CrossRef] [PubMed]

52. Purushottamachar, P.; Patel, J.B.; Gediya, L.K.; Clement, O.O.; Njar, V.C.O. First chemical feature-based pharmacophore modeling of potent retinoidal retinoic acid metabolism blocking agents (RAMBAs): Identification of novel RAMBA scaffolds. Eur. J. Med. Chem. 2012, 47, 412-423. [CrossRef] [PubMed]

53. Kumar, V.; Kumar, R.; Parate, S.; Yoon, S.; Lee, G.; Kim, D.; Lee, K.W. Identification of ACK1 Inhibitors as Anticancer Agents by using Computer-Aided Drug Designing. J. Mol. Struct. 2021, 1235, 1. [CrossRef]

54. Zeb, A.; Kim, D.; Alam, S.I.; Son, M.; Kumar, R.; Rampogu, S.; Parameswaran, S.; Shelake, R.M.; Rana, R.M.; Parate, S.; et al Computational Simulations Identify Pyrrolidine-2,3-Dione Derivatives as Novel Inhibitors of Cdk5/p25 Complex to Attenuate Alzheimer's Pathology. J. Clin. Med. 2019, 8, 746. [CrossRef] [PubMed]

55. Rampogu, S.; Parate, S.; Parameswaran, S.; Park, C.; Baek, A.; Son, M.; Park, Y.; Park, S.J.; Lee, K.W. Natural compounds as potential Hsp90 inhibitors for breast cancer-Pharmacophore guided molecular modelling studies. Comput. Biol. Chem. 2019, 83, 107113. [CrossRef]

56. Wu, L.; Viola, C.M.; Brzozowski, A.M.; Davies, G.J. Structural characterization of human heparanase reveals insights into substrate recognition. Nat. Struct. Mol. Biol. 2015, 22, 1016-1022. [CrossRef]

57. Messore, A.; Madia, V.N.; Pescatori, L.; Saccoliti, F.; Tudino, V.; De Leo, A.; Bortolami, M.; De Vita, D.; Scipione, L.; Pepi, F.; et al. Novel Symmetrical Benzazolyl Derivatives Endowed with Potent Anti-Heparanase Activity. J. Med. Chem. 2018, 61, 10834-10859. [CrossRef]

58. Pala, D.; Scalvini, L.; Elisi, G.M.; Lodola, A.; Mor, M.; Spadoni, G.; Ferrara, F.F.; Pavoni, E.; Roscilli, G.; Milazzo, F.M.; et al. New classes of potent heparanase inhibitors from ligand-based virtual screening. J. Enzym. Inhib. Med. Chem. 2020, 35, 1685-1696. [CrossRef] 
59. Pala, D.; Rivara, S.; Mor, M.; Milazzo, F.M.; Roscilli, G.; Pavoni, E.; Giannini, G. Kinetic analysis and molecular modeling of the inhibition mechanism of roneparstat (SST0001) on human heparanase. Glycobiology 2016, 26, 640-654. [CrossRef]

60. Pires, D.E.V.; Blundell, T.L.; Ascher, D.B. pkCSM: Predicting small-molecule pharmacokinetic and toxicity properties using graph-based signatures. J. Med. Chem. 2015, 58, 4066-4072. [CrossRef]

61. Awortwe, C.; Fasinu, P.S.; Rosenkranz, B. Application of Caco-2 cell line in herb-drug interaction studies: Current approaches and challenges. J. Pharm. Pharm. Sci. 2014, 17, 1-19. [CrossRef]

62. Lin, J.H.; Yamazaki, M. Role of P-glycoprotein in pharmacokinetics: Clinical implications. Clin. Pharmacokinet. 2003, 42, 59-98. [CrossRef]

63. Kim, S.; Chen, J.; Cheng, T.; Gindulyte, A.; He, J.; He, S.; Li, Q.; Shoemaker, B.A.; Thiessen, P.A.; Yu, B.; et al. PubChem in 2021: New data content and improved web interfaces. Nucleic Acids Res. 2021, 49, D1388-D1395. [CrossRef]

64. Weininger, D. SMILES, a Chemical Language and Information System: 1: Introduction to Methodology and Encoding Rules. J. Chem. Inf. Comput. Sci. 1988, 28, 31-36. [CrossRef]

65. Jiao, Z.; Hu, P.; Xu, H.; Wang, Q. Machine learning and deep learning in chemical health and safety: A systematic review of techniques and applications. J. Chem. Heal. Saf. 2020, 27, 316-334. [CrossRef]

66. Cadow, J.; Born, J.; Manica, M.; Oskooei, A.; Martínez, M.R. PaccMann: A web service for interpretable anticancer compound sensitivity prediction. Nucleic Acids Res. 2021, 48, W502-W508. [CrossRef]

67. Thirunavukkarasu, M.K.; Shin, W.H.; Karuppasamy, R. Exploring safe and potent bioactives for the treatment of non-small cell lung cancer. 3 Biotech. 2021, 11, 1-11. [CrossRef]

68. Liu, T.; Lin, Y.; Wen, X.; Jorissen, R.N.; Gilson, M.K. BindingDB: A web-accessible database of experimentally determined protein-ligand binding affinities. Nucleic Acids Res. 2007, 35, D198. [CrossRef]

69. Lin, S.-K. Pharmacophore Perception, Development and Use in Drug Design. Edited by Osman F. Güner. Molecules 2000, 5, 987-989. [CrossRef]

70. Lipinski, C.A.; Lombardo, F.; Dominy, B.W.; Feeney, P.J. Experimental and computational approaches to estimate solubility and permeability in drug discovery and development settings. Adv. Drug Deliv. Rev. 2001, 46, 3-26. [CrossRef]

71. Veber, D.F.; Johnson, S.R.; Cheng, H.Y.; Smith, B.R.; Ward, K.W.; Kopple, K.D. Molecular properties that influence the oral bioavailability of drug candidates. J. Med. Chem. 2002, 45, 2615-2623. [CrossRef]

72. Jones, G.; Willett, P.; Glen, R.C.; Leach, A.R.; Taylor, R. Development and validation of a genetic algorithm for flexible docking. J. Mol. Biol. 1997, 267, 727-748. [CrossRef] [PubMed]

73. Verdonk, M.L.; Cole, J.C.; Hartshorn, M.J.; Murray, C.W.; Taylor, R.D. Improved protein-ligand docking using GOLD. Proteins Struct. Funct. Genet. 2003, 52, 609-623. [CrossRef] [PubMed]

74. Kumar, V.; Parate, S.; Yoon, S.; Lee, G.; Lee, K.W. Computational Simulations Identified Marine-Derived Natural Bioactive Compounds as Replication Inhibitors of SARS-CoV-2. Front. Microbiol. 2021, 12, 583. [CrossRef] [PubMed]

75. Salsbury, F.R. Molecular dynamics simulations of protein dynamics and their relevance to drug discovery. Curr. Opin. Pharmacol. 2010, 10, 738-744. [CrossRef]

76. Kumar, R.; Kumar, V.; Lee, K.W. A computational drug repurposing approach in identifying the cephalosporin antibiotic and anti-hepatitis C drug derivatives for COVID-19 treatment. Comput. Biol. Med. 2021, 130, 104186. [CrossRef]

77. Abraham, M.J.; Murtola, T.; Schulz, R.; Páll, S.; Smith, J.C.; Hess, B.; Lindah, E. Gromacs: High performance molecular simulations through multi-level parallelism from laptops to supercomputers. SoftwareX 2015, 1-2, 19-25. [CrossRef]

78. Zhu, X.; Lopes, P.E.M.; MacKerell, A.D. Recent developments and applications of the CHARMM force fields. WIREs Comput. Mol. Sci. 2012, 2, 167-185. [CrossRef]

79. Zoete, V.; Cuendet, M.A.; Grosdidier, A.; Michielin, O. SwissParam: A fast force field generation tool for small organic molecules. J. Comput. Chem. 2011, 32, 2359-2368. [CrossRef]

80. Jaidhan, B.J.; Rao, P.S.; Apparao, A. Energy Minimization and Conformation Analysis of Molecules using Steepest Descent Method. Int. J. Comput. Sci. Inf. Technol. 2014, 5, 3525-3538.

81. Bussi, G.; Donadio, D.; Parrinello, M. Canonical sampling through velocity rescaling. J. Chem. Phys. 2007, 126, 14101. [CrossRef]

82. Parrinello, M.; Rahman, A. Polymorphic transitions in single crystals: A new molecular dynamics method. J. Appl. Phys. 1981, 52, 7182-7190. [CrossRef]

83. Darden, T.; York, D.; Pedersen, L. Particle mesh Ewald: An N·log(N) method for Ewald sums in large systems. J. Chem. Phys. 1993, 98, 10089-10092. [CrossRef]

84. Hess, B.; Bekker, H.; Berendsen, H.J.C.; Fraaije, J.G.E.M. LINCS: A linear constraint solver for molecular simulations. J. Comput. Chem. 1997, 18, 1463-1472. [CrossRef]

85. Humphrey, W.; Dalke, A.; Schulten, K. VMD: Visual molecular dynamics. J. Mol. Graph. 1996, 14, 33-38. [CrossRef]

86. Rifai, E.A.; Van Dijk, M.; Vermeulen, N.P.E.; Yanuar, A.; Geerke, D.P. A Comparative Linear Interaction Energy and MM/PBSA Study on SIRT1-Ligand Binding Free Energy Calculation. J. Chem. Inf. Model. 2019, 59, 4018-4033. [CrossRef]

87. Kumari, R.; Kumar, R.; Lynn, A. G-mmpbsa -A GROMACS tool for high-throughput MM-PBSA calculations. J. Chem. Inf. Model. 2014, 54, 1951-1962. [CrossRef] 